\title{
Estudio analítico y gráfico del dominio de los hormigones dosificados racionalmente
}

\author{
PEDro EnRique GRinszpan, Profesor titular de la E. Otto Kratise e I.I.s. (*) \\ Procesado y graficación con la colaboración de JORGE HCIso, M.M.d.o. \\ IV. REUNION TECNICA SOHRE TECNOLOGIA DEL HOIMIGON/A.A.T.H. \\ Mendoza + Noviembre 4 al 10 de 1979
}

\section{R E S U M E N}

Este trabajo pretende presentar un enfoque analítico del problema de la dosificación racional de hormigones y su interpretación en gráficos que evidencian la relación entre las distintas variables consideradas, las limitaciones impuestas y los factores económicos que condicionan este tema. El método propuesto permite el procesado por computadora, mediante la cual se obtiene un elevado número de resultados permitiendo su análisis e interpretación. Los resultados así obtenidos son una primera formulación del dosaje que se anticipa al indispensable estudio en laboratorio, brindan un punto de partida y resultan una solución para estudios preliminares y anteproyectos, suministrando para éstos los elementos de juicio necesarios para la elección de alternativas y estimación de costos. Tambien queda evidenciada la imposibilidad de realizar ciertas dosificaciones empíricas, tradicionalmente impuestas por el uso y las costumbres.

Por último se define un dominio de los hormigones dosificados racionalmente limitado a menos del dos por ciento de todas las composiciones representables.

\section{INTRODUCCION}

1.1. En el medio profesional no especializado se tiene por sencillo efectuar la composición de una mezcla fresca de hormigón y esa creencia no sería desacertada si la realización del hormigón fuese empírica en vez de racional y no implicase simultáneamente el cumplimiento de ciertos requisitos que aseguren su aptitud con relación a la construcción, las condiciones de uso y su vida útil posterior, satisfaciendo aspectos tan distintos como:

1. Inicialmente: Trabajabilidad (cohesión, docilidad, movilidad y colocabilidad).

2. Posteriormente: Resistencia mecánica a la compresión y en menor medida a la tracción (componentes, compacidad, relaciones ligante/agregado y agua/cemento).

3. Finalmente: Durabilidad (impermeabilidad, resistencia al desgaste y a las agresiones químicas).

(*) Profesor de Construcciones de Hormigón Armado, Estática y Estabilidad de las Construcciones, Proyectos de Hormigón Armado, y Tecnología del Hormigón y Práctica de Obra, de los cursos regulares y para técnicos graduados. 
1.1.2. Además presentándose soluciones alternativas que pueden cumplir, por igual, dichos requisitos, cabe condicionar la elección a criterios de economía.

1.2. Por consiguiente el enfoque racional de la dosificación debe incluir la consideración de:

- El destino del hormigón y las características de la obra.

- La resistencia prevista en el proyecto y los criterios de seguridad adoptados.

- Las características propias de los materiales empleados en la preparación (agua, cemento y agrega.dos).

- Las condic:ones propias de la elaboración y colocación.

- El costo de los componentes y el costo resultante de la mezcla.

1.3. El estudio analítico de las dosificaciones racionales, como primera aproximación del problema, implica la selección de las principales variables, determinar con suficiente aproximación sus leyes de variación y la interrelación entre ellas.

1.3.1. Dado el elevado número de estas variables y el complejo comportamiento de las mismas, la resolución analítica del problema, siempre y cuando los datos sean suficientemente precisos, sólo nos brinda una muy buena aproximación a la solución definitiva que dependerá de la tarea de laboratorio que mediante "pastones de prueba" permitirá corroborar los valores obtenidos y efectuar los ajustes necesarios en forma experimental.

1.3.2. Sin embargo la propuesta analítica, aun cuando no es suficiente, brinda el beneficio de un punto de partida para la tarea experimental y por otra parte una solución para los estudios preliminares y anteproyectos que generalmente por razones de tiempo y oportunidad no admiten acudir a los recursos del laboratorio, suministrando los juicios básicos para la elección de materiales, dosificaciones y estimación de costos, en relación con lo último especialmente cuando se trata de estudios para concursos y licitaciones.

\section{CONSIDERACIONES GENERALES}

En este estudio se consideran las sigu:entes variables agrupadas, según se expone a continuación, en principales, complementarias y secundarias.

\subsection{Variables principales}

Constituidas por los componentes básicos del hormigón, cuyas cantidades se expresan por unidad de volumen $\left(1 \mathrm{~m}^{3}\right)$ de mezcla fresca de hormigón, expuestas en peso $(p)$ o en volumen absoluto o real $(v r)$ :

A: Agua de mezclado.

$C$ : Cemento.

I: Agregados inertes, constituidos por:

$F$ : Finos.

$G$ : Gruesos.

\subsection{Variables complementarias}

Caracterizan las propiedades de la mezcla fresca y endurecida, son:

$x$ : Relación agua/cemento expresada en peso $x=(A / C) p$.

6 
$m$ : Módulo granulométrico o de finura de Abrams correspondiente a la composición total del agregado $m=$ Sum. $R_{1, r} / 100$.

$y$ : Coeficiente de variación de la cons.stencia de la mezcla fresca en función del "asentamiento" determinado mediante el ensayo del tronco cono de Abrams.

\subsection{Variables secundarias}

Expresan el costo relativo de los componentes por unidad de volumen absoluto o real (vr) de los mismos considerando como unitario el correspondiente al agregado más fino (la arena fina $F f)$ :

$I U(A)=0,1$ para el agua (costo de uso).

$I U(C)=16$ para el cemento.

$I U(E f)=$ para la arena fina argentina $\left(F_{1}\right): 1$.

$I U(F g)=2$ para la arena gruesa tipo oriental $(F 2)$.

$I U(G r)=4$ para el agregado grueso redondeado $(C R)$.

$I U(G a)=3,6$ para el agregado grueso angular $(P P)$.

$$
\begin{aligned}
& (G r, \text { ó, } G a=G 4) \\
& (F 1+F 2=F 3) \\
& (F 3+G 4=I 5)
\end{aligned}
$$

\section{ANALISIS}

3.1. Iniciamos este planteo expresando la composición del hormigón mediante la siguiente ecuación:

$$
H=(A+C+F+G)+(a+q),
$$

excluyendo el término complementario formado por:

$a=$ aire (accidental y/o intencional).

$q=$ aditivos: tendremos con

$$
\begin{gathered}
F+G=I \text { (agregado inerte total) } \\
H=A+C v r+I v r=1.000 \text { litros transformable en } \\
H=A+\left(1 / P_{\mathrm{eC}}\right) \cdot C p+\left(1 / P_{\mathrm{e} I}\right) \cdot I p=1.000
\end{gathered}
$$

con los componentes en peso y para los pesos específicos medios $(\mathrm{Pe})$ de ambos materiales resulta:

$$
H=A+0,32 \cdot C p+0,38 \cdot I p=1.000
$$

Además se considera la expresión de Abrams (*) para la determinación del agua de mezclado en función de la requerida por el cemento y también por el agregado según sus características:

$$
A_{0}=0,23 \cdot C p+k \cdot(0,16-m / 50) \cdot I p
$$

donde $A_{0}$ es la cantidad de agua correspondiente a una mezcla de asentamiento normalizado de $3 "=$ aprox. $71 / 2 \mathrm{~cm}, k s /$ tipo de agregado $=(1, p / C . R \text {. ó } 1,2 \text { p/P.P. })^{\prime}$ y definiendo

(*) “Control y ensayo de cementos, morteros y hormigones". M. Venuat y M. Papadakis, ed. Urmo, Bilbao 1966; pg. 253. Edición original en lengua francesa publ. p/Eyrolles. 
$A=y \cdot A_{0}$ la cantidad de agua correspondiente a consistencias de distintos asentamientos $(\neq 7,5)$ con " $y$ " como el coeficiente de variación en función del asentamiento, resulta:

$$
A_{0}=A / y=0,23 \cdot C p+\frac{2 \cdot k}{100} \cdot(8-m) \cdot I p
$$

Si addemás sustituimos en dicha ecuación $A=x \cdot C p$, e igualmente en la primera, tendremos:

$$
\begin{gathered}
(x+0,32) \cdot C p+(0,38) \cdot I p=1.000 \\
(x / y-0,23) \cdot C p+2 k / 100 \cdot(8-m) \cdot I p=0
\end{gathered}
$$

Sistema que resuelto permite obtener los valores de:

con

$$
C p=N c / D ; I p=N i / D ; \text { con }
$$

$$
\begin{gathered}
N c=20 \cdot k \cdot(8-m) \\
N i=1.000 \cdot(x / y-0,23) \\
D=(x+0,32) \cdot[0,02 \cdot k \cdot(8-m)]+0,38 \cdot(x / y-0,23)
\end{gathered}
$$

siendo:

$$
C v r=0,32 \cdot C p \quad ; \quad I v r=0,38 \cdot I p
$$

3.2. En estas expresiones el coeficiente " $y$ " de variación del contenido de agua con relación a la consistencia según el ensayo de asentamiento mediante el tronco cono de Abrams, resulta de la curva experimental establecida en el trabajo sobre dosificaciones realizado por García Balado (*) a partir de la cual se puede formular una ecuación parabólica ajustada a los siguientes valores: $A S \cdot d \cdot(y)$.

$$
A S \cdot(\mathrm{cm})=d=2,5(0,90) \quad ; \quad 7,5(1) \quad ; \quad 27,5(1,20)
$$

resulta entonces la siguiente expresión

$$
y=1,2025-\left(\frac{30-d}{50}\right)^{2}
$$

3.3. En cuanto al módulo granulométrico " $m$ " de la composición total del agregado, éste queda vinculado a una curva granulométrica limitada por el tamaño mayor nominal " $D$ " mediante funciones como las siguientes:

3.3.1. Parábola de Gessner generalizada $\left({ }^{* *}\right)$ expresable mediante la siguiente ecuación

$$
m=n-\frac{1-(0,65 \text { a } 0,85)^{\mathrm{n}}}{1-(0,65 \text { a } 0,85)}
$$

en la cual $c=0,65$ a 0,85 define el grado de la parábola, con $c=0,7$ se tiene la solución tradicional correspondiente a la parábola cuadrática de Fuller-Gessner, " $n$ " es el número de orden del tamiz correspondiente al tamaño mayor nominal " $D$ " en la serie normalizada de tamices usada por Abrams, y es simultáneamente el módulo granulométrico propio de dicho tamiz.

\footnotetext{
(*) "Método para la dosificación de hormigones". J. F. García Balado; publ. n.o 42-E.4 del I.C.P.A., Bs.As/v. Lámina 10, pg. 54.

(**) “Interpretación y medición comparada de lineas granulométricas continuas”. P. E. Grinszpan, Bs.As.
} 
3.3.2. Ecuaciones de M. Sabesinsky-Felperin $\left({ }^{*}\right)$, vinculan " $m$ " con " $D$ " para cada clase de agregado:

$$
\begin{aligned}
& m=2,28+2,248 \cdot \operatorname{Lg} D \quad \text { para agregado redondeado, y } \\
& m=1,85+2,514 \cdot \operatorname{Lg} D \quad \text { para agregado angular, con " } D \text { " en milímetros. }
\end{aligned}
$$

Estas ecuaciones son susceptibles de reducirse a una única del siguiente formato:

$$
m=2,28 \times 0,811^{\mathrm{a}}+2,248 \times 1,118^{\mathrm{a}} \cdot \operatorname{Lg} D
$$

con el exponente

$$
\text { " } a "=0 \text { (p/C.R.) ó } 1 \text { (p/P.P.) }
$$

esta ecuación puede simplificarse en:

aprox.

$$
m_{1}=2 \times 1,13 \cdot\left(0,8^{\mathrm{a}}+1,13^{\mathrm{a}} \cdot \operatorname{Lg} D\right)
$$

transformándose finalmente en:

aprox. $\quad m=2 \times 1,13 \cdot\left[0,8^{\mathrm{a}}+1,13^{\mathrm{a}} \cdot(0,3 \cdot n-1,13)\right]$

3.3.3. De lo anterior resultan composiciones ternarias constituidas por dos clases de finos $(F 1, F 2)$ y una de grueso $(G 4)$ con:

$$
m_{3}=P_{1} \cdot m_{1}+P_{2} \cdot m_{2} \text { para la mezcla de finos (F3) }
$$

finalmente

$$
m=m_{5}=P_{3} \cdot m_{3}+P_{4} \cdot m_{4}=P_{1} \cdot P_{3} \cdot m_{1}+P_{2} \cdot P_{3} \cdot m_{2}+P_{4} \cdot m_{4}
$$

para la composición total de finos y grueso, donde $m_{\mathrm{i}}$ es el módulo granulométrico y $p_{\mathrm{i}}$ es la proporción respecto a la unidad $P_{5}=1$. De este modo se puede establecer las proporciones $P_{1}(F 1), P_{2}(F 2)$, y $P_{4}(G 4)$ en las que se reparte el total $P_{5}(15)$ del agregado global identificado con $m=m_{5}$ a los efectos que se apreciará más adelante.

3.4. Los criterios de economía se relacionan al costo de los materiales utilizados en la elaboración de la mezcla fresca de hormigón, mediante la aplicación de los índices de costos unitarios antes enunciados (ver 2.3.) resultando un índice de costo (I.C.) por $\mathrm{m}^{3}$ de hormigón fresco, como sigue:

I.C. $\left(p / m^{3}\right)=[0,1 \cdot A+16 \cdot C+F 1+2 \cdot F 2+(4 \cdot G r$ ó 3,6 Ga $)] p / C R$ ó PP

3.4.1. Teniendo en cuenta que dos mezclas de igual (I.C.) índice de costo pueden corresponder a hormigones de distinta resistencia obtenible mediante la ecuación $\left(^{*}\right)$ siguiente:

$$
R^{\prime} h=(3,1 \text { a } 3,7) \cdot R^{\prime} m /(25)^{x}
$$

resulta de interés establecer el costo en relación a la resistencia determinada, es decir un índice ponderado (I.P.): I.C.P. $=$ costo $p / \mathrm{m}^{s}$ : Resistencia $\left(R^{\prime} h\right)$, para ello se adopta como índice de costo ponderado la siguiente relación:

$$
\text { I.C.P. }=\left(25^{\times} / 10\right) \cdot \text { I.C. }
$$

en el caso del agregado angular este índice; queda gravado en un veinte por ciento como modo de considerar las dificultades inherentes al empleo de esta clase de agregado en las obras corrientes.

\footnotetext{
(*) "Proyecto de Hormigones", ed. Nigar. Bs.As. 1973, cap. 4/pg. 104.

(**) Ecuación debida al Ing. G. N. Burgoa, consultar "Informe n.o 1 de la Subcomisión de Nornas de la A.A.T.H." y sus antecedentes al 27.09/77; IIIa. Rcunión Técnica A.A.T.H., Concordia 1977.
} 


\section{PROCESADO}

4.1. Considerando el conjunto de ecuaciones (I) a (IX) del apartado anterior 3. es sencillo organizar un programa para el procesado en microcomputadores de la clase "H.P." o "T.I." y desarrollar los resultados de dosificaciones seriadas. En este caso se ha aplicado un programa diseñado para un equipo de la clase "T.I. 59".

4.2. Se ha planificado distintas series para el siguiente conjunto de datos:

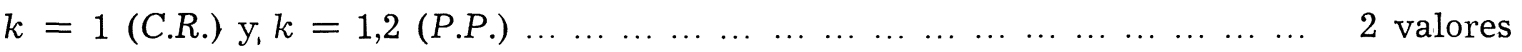

$$
\begin{aligned}
& x=0,45-0,50-0,52-0,54-0,56-0,58-0,60-0,65-0,70-0,80-0,90 \text { y } 1,00 \ldots 12 \text { valores } \\
& y=0,950-1,000-1,025-1,050-1,075-1,100 \text { y } 1,125 \ldots \ldots \ldots \ldots \ldots \ldots \ldots \quad 7 \text { valores } \\
& \text { correspondiente aproximadamente a }
\end{aligned}
$$$$
d=5-7,5-9-10-12-14 \text { y } 16 \mathrm{~cm} \text { de asentamiento; }
$$

las siguientes granulometrías:

$m=4,50-4,75-5-5,25-5,50-5,75$ y 6

obteniéndose un total de dosificaciones:

total de dosificaciones $=2 \times 12 \times 7 \times 7=1.176$, para las cuales se calcula:

I : Aregado total en peso $(P)$ y en vol. absoluto $(v r)$.

$C$ : Cemento en peso $(P)$ y en vol. absoluto $(v r)$.

$A$ : Agua en volumen $\left(\mathrm{m}^{3}\right)$.

$\alpha$ : Relación agregado/cemento en volumen aparente $=(I / C) v a$.

Gvr: Agregado grueso en volumen absoluto.

Fvr : Agregado fino total en volumen absoluto.

$\% F$ : Porcentaje de agregado fino en peso.

F2vr: Arena gruesa en volumen absoluto.

F1vr: Arena fina en volumen absoluto.

IC : Indice de costo relativo $P / \mathrm{m}^{3}$ de hormigón fresco.

ICP : Indice de costo ponderado relativo, $P /$ unidad de volumen y por $P /$ unidad de resistencia.

4.3. Por razones de espacio se adjuntan solamente algunas de las planillas del procesado con la mera finalidad de ejemplificar el procedimiento seguido. El conjunto de los resultados obtenidos se ha interpretado gráficamente a efectos de evidenciar las relaciones entre las distintas variables y sus leyes de comportamiento.

\section{INTERPRETACION GRAFICA}

\subsection{Procedimiento gráfico adoptado}

Se ha encontrado particularmente conveniente utilizar el procedimiento gráfico triangular ideado por Feret (para los materiales cementantes) que permite trabajar con tres variables simultáneamente $(*)$, número que coincide con el de las incógnitas principales (ver 2.1.) consideradas en este estudio y que son los componentes básicos del hormigón ( $A$, $C, I)$.

Sobre los tres lados del triángulo equilátero se representa de 0 a 1.000 litros el volumen absoluto (vr) de los componentes, de tal modo que cualquier punto del gráfico represen-

(*) “Tecnologia del Hormigón”. Julio R. Castiñeiras, ed. del C.E.I. Bs.As. 1941; v./ Cap. I, pg. 24; Represent. triang. ... 
ta una composición en la cual los tres términos indicados suman 1.000 litros correspondientes a la unidad de volumen $\left(1 \mathrm{~m}^{3}\right)$ adoptada para la mezcla fresca de hormigón sin aire ni aditivos. Considerando que las cantidades de agua $(A)$ y de cemento $(C)$ son menores de 500 litros y que la cantidad de agregado Inerte $(I)$ es superior a los 500 litros, puede limitarse el gráfico a un triángulo reducido a una cuarta parte del total; de este modo se amplía la escala de la representación al sector utilizado (ver gráficos). En los gráficos se ha diferenciado siempre las dos clases de agregados, el redondeado con $k=$ $=1$, y el angular con $k=1,20$.

\subsection{Los dosajes y su representación}

5.2.1. Las figuras 1 y 3 muestran para dosificaciones con $x=0,70$ cómo todos los valores se encuentran alineados sobre una recta que nace en el vértice inferior derecho $(A=0$; $I=1.000)$ determinando un ángulo $\varnothing$ con la base; su ecuación según puede demostrarse fácilmente es:

$$
\operatorname{Tg} \varnothing=F(x)=(C / 2) \cdot \operatorname{Tg} 60 /[(C / 2)+A]=1,732 /(1+6,25 \cdot x)
$$

siendo:

$$
0 \leqslant \varnothing \leqslant 60^{\circ} \quad \text { con } \quad \text { inf. } \geqslant x \geqslant 0
$$

para

$$
\begin{aligned}
& x=0,40 ; \varnothing=26^{\circ} 19^{\prime} 43^{\prime \prime}, 8 \\
& x=1,00 ; \varnothing=13^{\circ} 26^{\prime} 09^{\prime \prime}, 4
\end{aligned}
$$

Sobre esta recta se encuentran los siete puntos para un mismo valor de " $y$ " (consistencia constante) y valores de " $m$ " de 4,50 a 6,00; para valores crecientes de " $y$ " (mezclas de mayor fluidez) el segmento constituido por los siete puntos de " $m$ " se desplaza hacia la izquierda. Entre los puntos $(y=1,125 ; m=4,50)$ e $(y=0,950 ; m=6,00)$ se encuentran todas las dosificaciones de igual relación agua/cemento " $x$ ".

5.2.2. Las figuras 2 y 4 muestran para los distintos valores de $x=0,40$ hasta $x=1,00$ la disposición de los segmentos antes mencionados (5.2.1.) en forma de abanico sobre un haz de rectas con origen en el vértice inferior derecho del triángulo.

Uniendo los puntos de $m=4,50$ y de $m=6,00$ respectivamente, se definen cuadriláteros que abarcan la zona de todos los valores comprendidos entre los límites elegidos.

5.2.3. La figura 5 expone la superposición parcial de los cuadriláteros para $k=1,00 \mathrm{y}$ $k=1,20$ que corresponde a los puntos de todos los dosajes procesados (1.176 casos) para los distintos valores de todas las variables complementarias:

$$
\begin{aligned}
K & =1,00 / 1 ; 20 \\
x & =0,40 / 1,00 \\
m & =4,50 / 6,00 \\
y & =0,95 / 1,125
\end{aligned}
$$

Puede observarse en la figura la ubicación de las dosificaciones correspondientes a mezclas con relación $x=0,50$.

La figura resultante nos enseña el dominio de todas las dosificaciones entre los límites expresados y su área representa aproximadamente el diez por ciento $(10 \%)$ de la superficie del triángulo reducido, valor que se reduce a la cuarta parte cuando queda referido 
al triángulo total $(\leqslant 3 \%)$ que a su vez representa al cien por ciento $(100 \%)$ de las combinaciones numéricas de todas las composiciones $(A+C+I)$ practicables e impracticables, vemos que menos del tres por ciento del total corresponde al dominio de las practicables, convenientes o solamente aceptables. Este dominio es susceptible de sufrir otras restricciones que limitan aún más posibilidades técnicamente factibles de ser consideradas, como se verá a continuación.

\subsection{Relación agregados/cemento}

5.3.1. Siendo muy frecuente la expresión del dosaje mediante la relación entre las cantidades de agregado inerte y del cemento, relación sumamente imprecisa cuando se establece en función de los volúmenes aparentes $(v a)$ de los materiales, pero lamentablemente impuesta por la costumbre, por ello resulta particularmente interesante introducir dicha relación en los gráficos.

$$
\alpha=I v a / C v a=\frac{I v r \cdot(P e / P u) i}{C v r \cdot(P e / P u) c}=(I v r / C v r) \cdot \gamma
$$

Vienda las figuras 6 y 7 puede observarse que las dosificaciones que corresponden a un mismo valor de " $\alpha$ " se encuentran sobre una recta que tiene su origen en el vértice inferior izquierdo ( $A=1.000 ; C=0)$ determinando un ángulo " $j$ " con la base; su ecuación, según es fácil demostrar, es:

$\operatorname{Tg} j=F(x)=(C / 2) \cdot \operatorname{Tg} 60 /[(C / 2)+I]=1,732 /(1+2 \cdot \alpha / \gamma)=1,732 /(1+2,88 \cdot \alpha)$

para $\gamma=1 / 1,44$

En dichos gráficos se observa la intersección de los haces de rectas para valores de " $\alpha$ " desde 4 hasta 7 , y para valoresi de " $x$ " de 0,50 hasta 1,00 .

5.3.2. Indicando los valores que se encuentran en los dominios de los cuadriláteros de dosajes para $k=1,0$ y $k=1,20$ (agregado redondeado y angular respectivamente) se visualiza las resistencias obtenibles (en función de " $x$ ") para distintas proporciones dentro de los límites plausibles.

Puede observarse que para valores bajos de " $\alpha$ " se tienen mezclas de elevado contenido de pasta cementicia y agregado de bajo módulo granulométrico total tendiendo a pasar de los hormigones a los morteros.

5.3.3. De las ecuaciones (X) y (XII) se obtienen las siguientes que permiten vincular " $\alpha$ " con " $x$ ", y ambos valores con " $C$ " contenido de cemento de la mezcla:

$$
\begin{gathered}
6,25 \cdot x=(1,732 / \operatorname{Tg} \varnothing)-1 \\
2,88 \cdot \alpha=(1,732 / \operatorname{Tg} j)-1 \\
\operatorname{Tg} \varnothing / \operatorname{Tg} j=(1+2,88 \cdot \alpha) /(1+6,25 \cdot x)
\end{gathered}
$$


y mediante éstas finalmente:

$$
\operatorname{Tg} \varnothing / \operatorname{Tg} j=[1.000 /(A+C / 2)]-1=(1+2,88 \alpha) /(1+6,25 x)
$$

de donde resulta:

$$
1.000 / C=\{[(1+2,88 \alpha) /(1+6,25 x)]+1\} \cdot[(x / 0,32)+0,5]
$$

con la cual se produjo el gráfico de la figura 8 en el que se fijaron cuatro niveles de contenido de cemento:

$$
C=400,350,300 \text { y } 250 \mathrm{~kg}\left(p / m^{3}\right)
$$

Se obtuvieron los valores de " $\alpha$ " (=Iva/Cva) para las rectas de " $x$ " $(=A / C p)$ de 0,50 a 1,00. Fijados los límites (ver segmentos de trazos cortados) para mezclas de máximo contenido de agua ( $A$, para: $k=1,2 ; y=1,125 ; m=4,50)$ y de mínimo contenido de agua ( $A$, para: $\left.k=1 ; y=0,95 ; m_{1}=6\right)$.

5.3.3.1. Este gráfico es particularmente ilustrativo pues en él puede observarse que para un contenido de cemento $C=300 \mathrm{~kg}$ la relación $\alpha=(i / C)$ va varía entre $4,8(p / x=0,80)$ y $5,4(p / x=0,50)$, mientras que para $\alpha=6(p / x=0,90)$ se encuentra con un contenido de cemento $C=250 \mathrm{~kg}$; este valor de " $\alpha$ " se encuentra también para $x=0,40$ con $C=$ $=290 \mathrm{~kg}$, y para $x=0,30$ con $C=295 \mathrm{~kg}$, fuera de los límites del dominio marcado precedentemente. Se comprueba así la imposibilidad de cumplir con la dosificación empírica (1:6) $v a$ para $C=300 \mathrm{~kg}$ que sin embargo tan frecuentemente se incluye en las especificaciones técnicas con ignorancia de los parámetros fundamentales de una dosificación racional.

5.3.3.2. En las condiciones consideradas en el párrafo anterior se mencionaron dosificaciones sin diferenciar la consistencia de la mezcla (coeficiente " $y$ ") ni la granulometría del agregado (módulo " $m$ "), de incluir éstas y considerando:

$$
\alpha_{\mathrm{p}}=(I / C) p=I v a \cdot P u I / C v a \cdot P u C=(P u I / P u C) \cdot \alpha=(1,20 \text { a } 1,30) \cdot \alpha_{\mathrm{va}}
$$

Se puede transformar la ecuación (I) en:

$$
(0,32+x) \cdot C p+0,38 \cdot\left(\alpha_{\mathrm{p}} \cdot C p\right)=\left(0,32+x+0,38 \cdot \alpha_{\mathrm{p}}\right) \cdot C p=1.000
$$

y la ecuación (II) en:

$$
\begin{aligned}
& (x / y-0,23) \cdot C p-\frac{2 \cdot k}{100} \cdot(8-m) \cdot\left(\alpha_{\mathrm{p}} \cdot C p\right)= \\
& \quad=\left[x / y-0,23-\frac{2 \cdot k}{100} \cdot(8-m) \cdot \alpha_{\mathrm{p}}\right] \cdot C p=0
\end{aligned}
$$

siendo $C p \neq 0$ de donde resulta:

$$
x / y=0,23+\frac{2 \cdot k}{100} \cdot(8-m) \cdot \alpha_{p}
$$


o también:

$$
m=8-100 \cdot(x / y-0,23) / 2 \cdot k \cdot \alpha_{\mathrm{p}}
$$

En la ecuación $(\mathrm{XX})$ para valores:

$$
(m / \alpha / k) \text { de }(4,5 / 1,25 \cdot 7 / 1,2) \text { hasta }(6 / 1,25 \cdot 4 / 1)
$$

varía $x / y$ de 0,96 a 0,43 , mientras que en la ecuación (XXI) para valores:

$$
(x / y / k / \alpha) \text { de }(0,8 / 0,95 / 1 / 1,25 \times 4) \text { a }(0,4 / 1,125 / 1,2 / 1,25 \times 6)
$$

varía el módulo $m$ de 1,99 a 7,3.

Estos valores abarcan extremos dentro de los cuales cabe encontrar las soluciones convenientes; los valores bajos de " $\alpha$ " corresponden naturalmente a valores bajos de " $m$ " e indican soluciones dentro del ámbito de los morteros.

5.3.4. La figura 9 representa la reducción del dominio entre los límites de $\alpha=4,0$ hasta $\alpha=7,0$ que de este modo se circunscribe aproximadamente al cincuenta por ciento del área del cuadrilátero original, quedando el hexágono irregular que se muestra rayado en la lámina.

\subsection{Relaciones económicas/índices de costo}

5.4.1. Las figuras $10,11,12(\mathrm{p} / \mathrm{k}=1)$ y las $13,14,15(\mathrm{p} / \mathrm{k}=1,20)$ muestran las intersecciones de las rectas correspondientes a soluciones con $x=$ cte. y con $m=$ cte.; los puntos correspondientes a las combinaciones de

$$
\begin{aligned}
x & =0,50-0,60-0,70-0,80-0,90-1,00 \\
m & =4,50-5,00-5,50-6,00
\end{aligned}
$$

pertenecen a las veinticuatro soluciones para las cuales se han tabulado los índices de costo por unidad de volumen (I.C.) y ponderado por resistencia (I.C.P.). Estas tablas acompañan a los seis gráficos correspondientes a distintas combinaciones de agregados y fluidez de las mezclas.

5.4.2. Observando estos valores se comprende las leyes de variación de costos de las dosificaciones según su situación en el cuadrilátero constituido por los puntos registrados y todos los intermedios restantes. Nótese que los correspondientes a los cuatro vértices corresponden a distintos niveles de contenido de cemento $(C)$ (ver líneas horizontales) que varían entre $35 l(110 \mathrm{~kg})$ y 179 l $(560 \mathrm{~kg})$.

5.4.3. Los valores fueron obtenidos mediante la aplicación de las ecuaciones (VII) y (IX) y las conclusiones que se desprenden de su estudio se han graficado en la figura 16, en donde las flechas indican el sentido de variación de los costos según cada variable. La figura se realizó para un módulo constante $m=5,500$ (valor medio) y agregado angular con $k=1,20(P . P$.

5.4.4. La figura 17 analiza las variaciones de costos por medio de los índices mencionados, para ambas clases de agregado grueso y distintos módulos granulométricos, pero a diferencia de la anterior, para mezclas de consistencia constante, se acota los límites de variación de cada variable indicando en qué santido crece. 
5.4.5. En ambas figuras ( 16 y 17) puede observarse que en cada dirección indicada, los valores del I.C. y el I.C.P. crecen en sentidos antagónicos; resulta evidente que ambos índices (I.C. e I.C.P.) sólo pueden crecer simultáneamente sobre las rectas de " $x$ " $(A / C=$ $=$ constante).

\section{CONCLUSIONES}

A lo largo de los cinco capítulos anteriores se ha efectuado un análisis y síntesis del problema: de la dosificación racional de los hormigones, considerando sus variables más significativas y leyes de variación de éstas apoyadas en los resultados habitualmente aceptados de la investigación experimental, con las limitaciones expuestas en "1.3.".Dichas variables fueron ordenadas en el Capítulo 2, estableciéndose su importancia; para las secundarias (de costos) se fijaron valores circunstanciales que no afectan las interpretaciones y conclusiones de carácter general. En el Capítulo 3 se realizó un planteo analítico que conduce a un sistema de ecuaciones mediante las cuales se alcanzan resultados en forma directa para cada conjunto de condiciones impuestas. La obtención posible de estos resultados mediante un procesado por computadora es consecuencia directa de lo anterior y queda expuesto en el Capítulo 4 de este trabajo. En el Capítulo 5 se vuelca dicho conjunto sobre representaciones gráficas, cuya interpretación revela importantes relaciones y acota el dominio posible de las dosificaciones a menos del dos por ciento de todas las combinaciones representables e introduce, además, los elementos de análisis económico necesarios para efectuar una selección entre las posibles alternativas. 


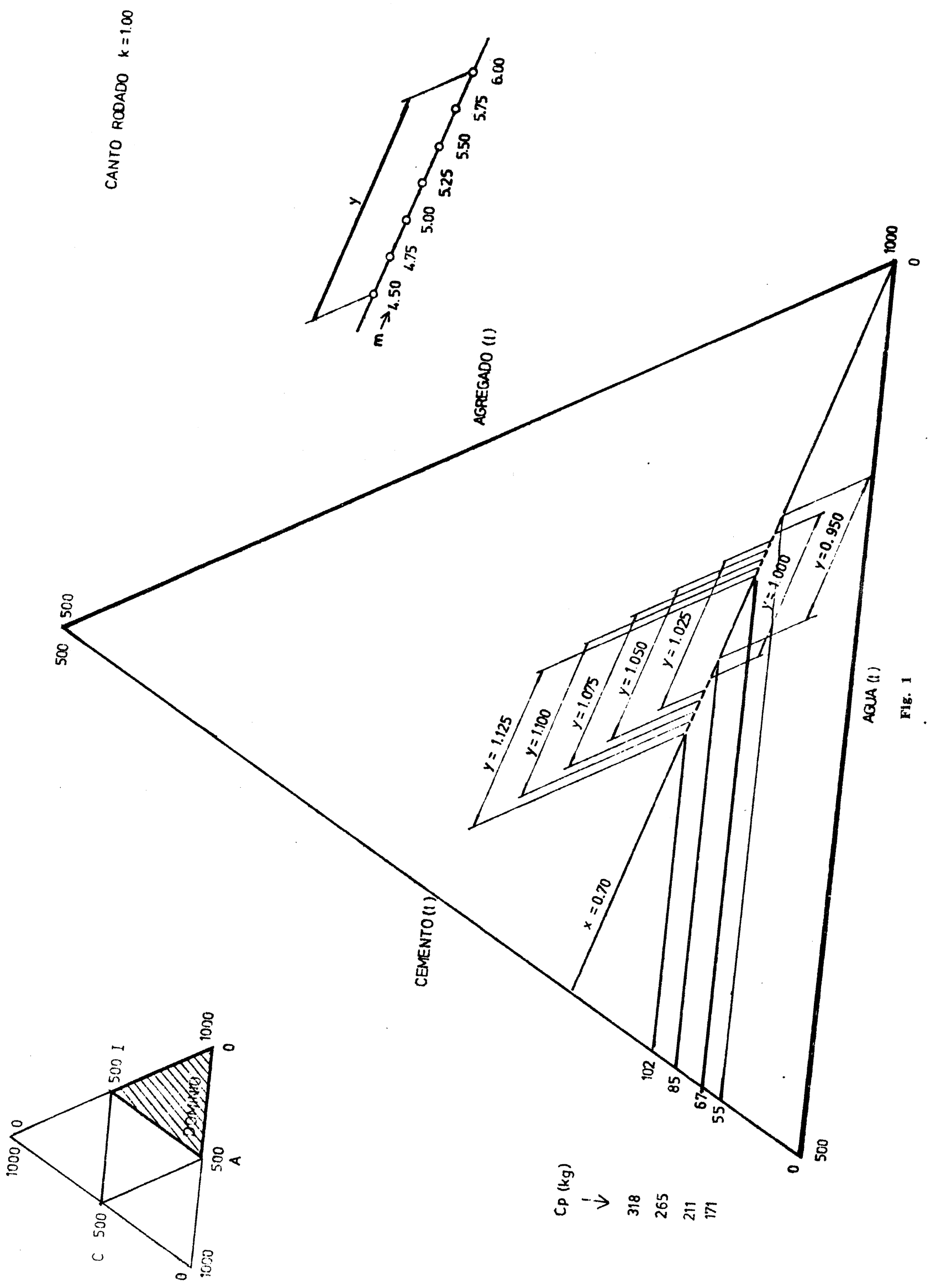




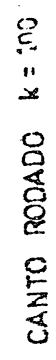
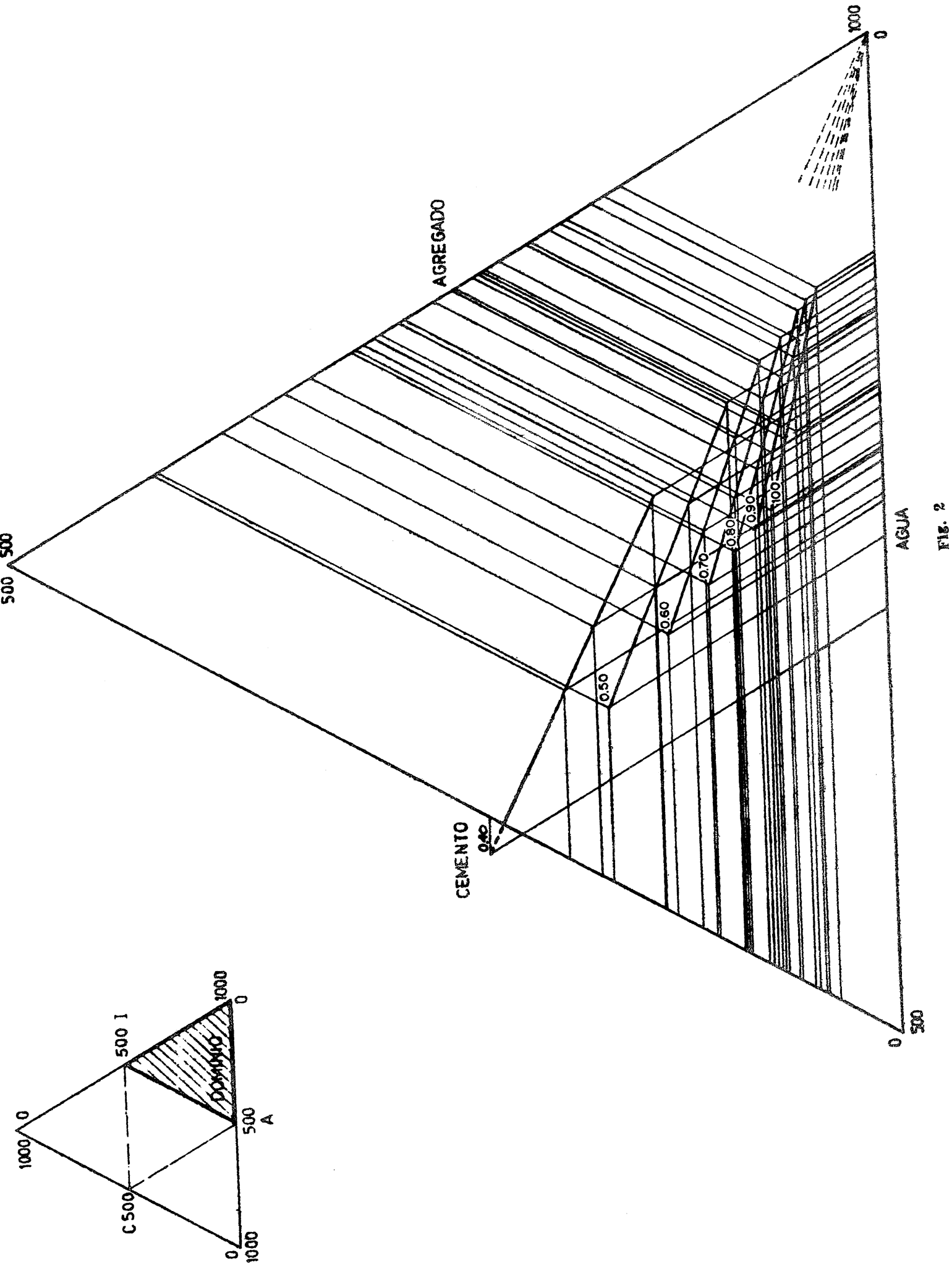

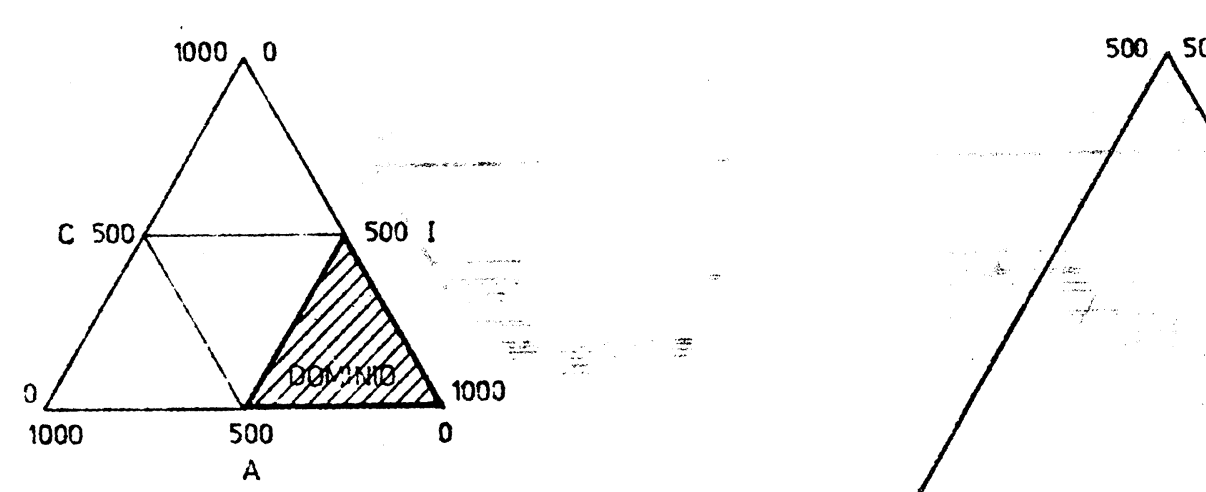

IEDRA PARTIDA $k=1.20$

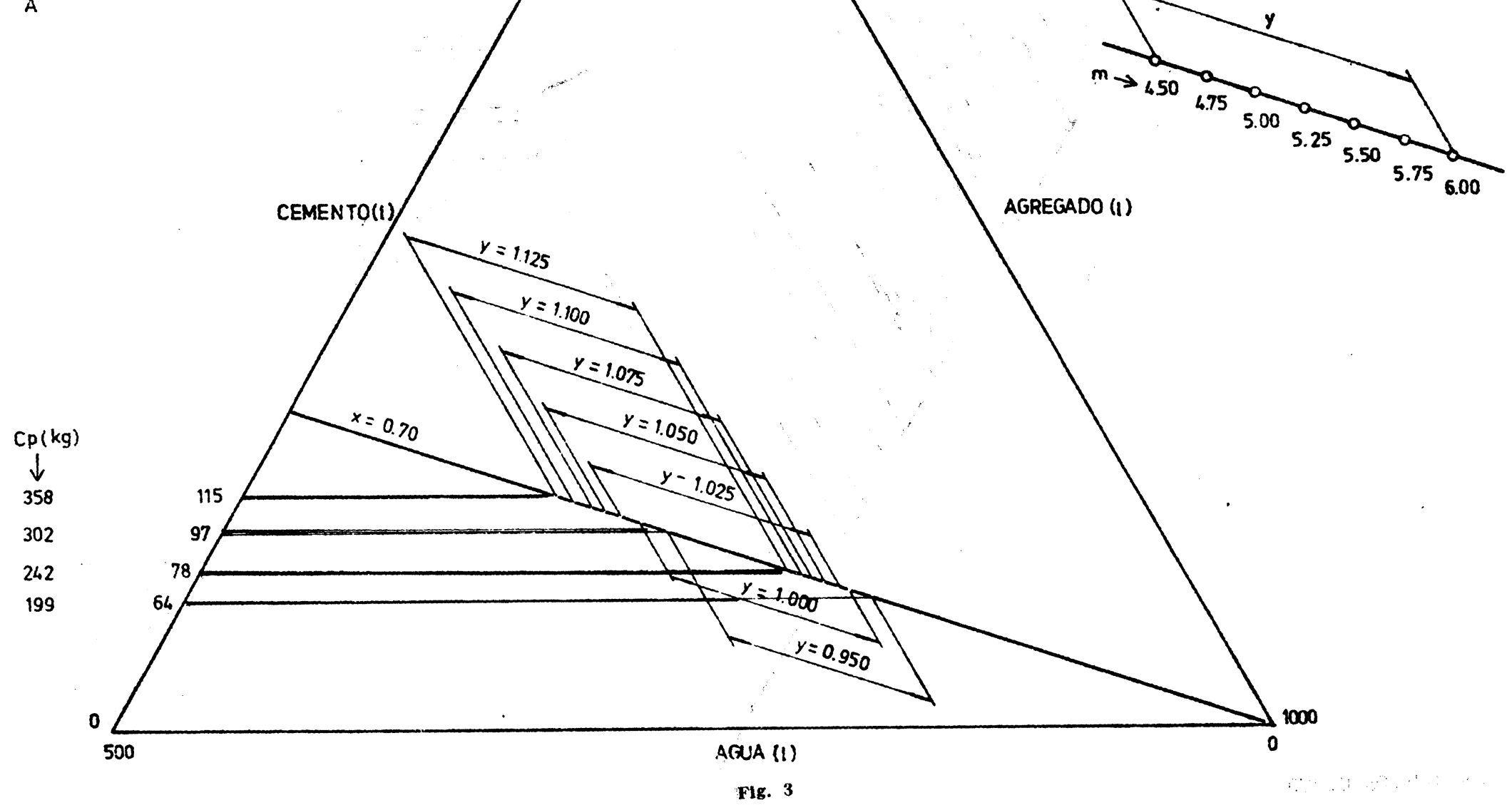




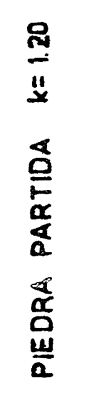
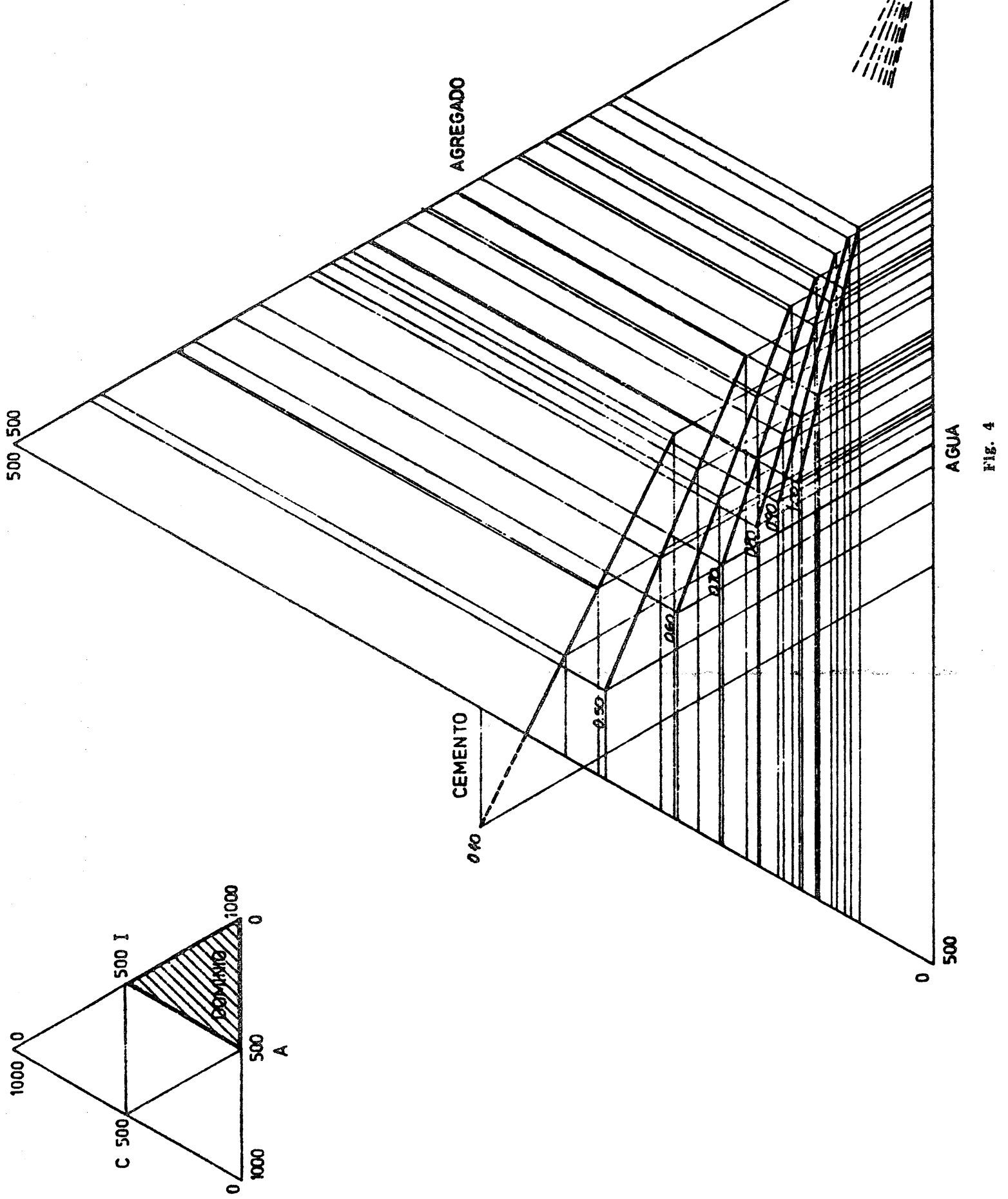


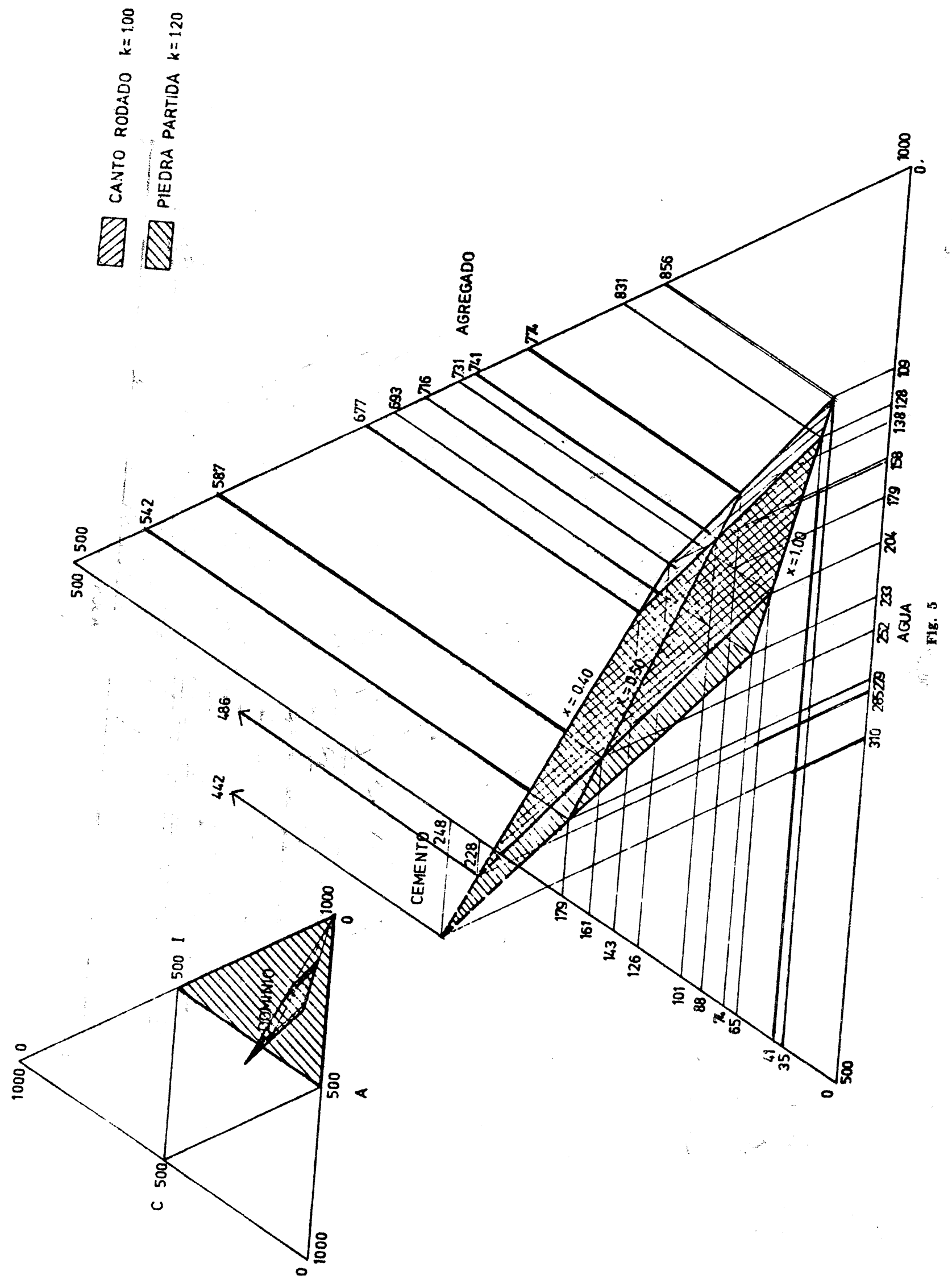




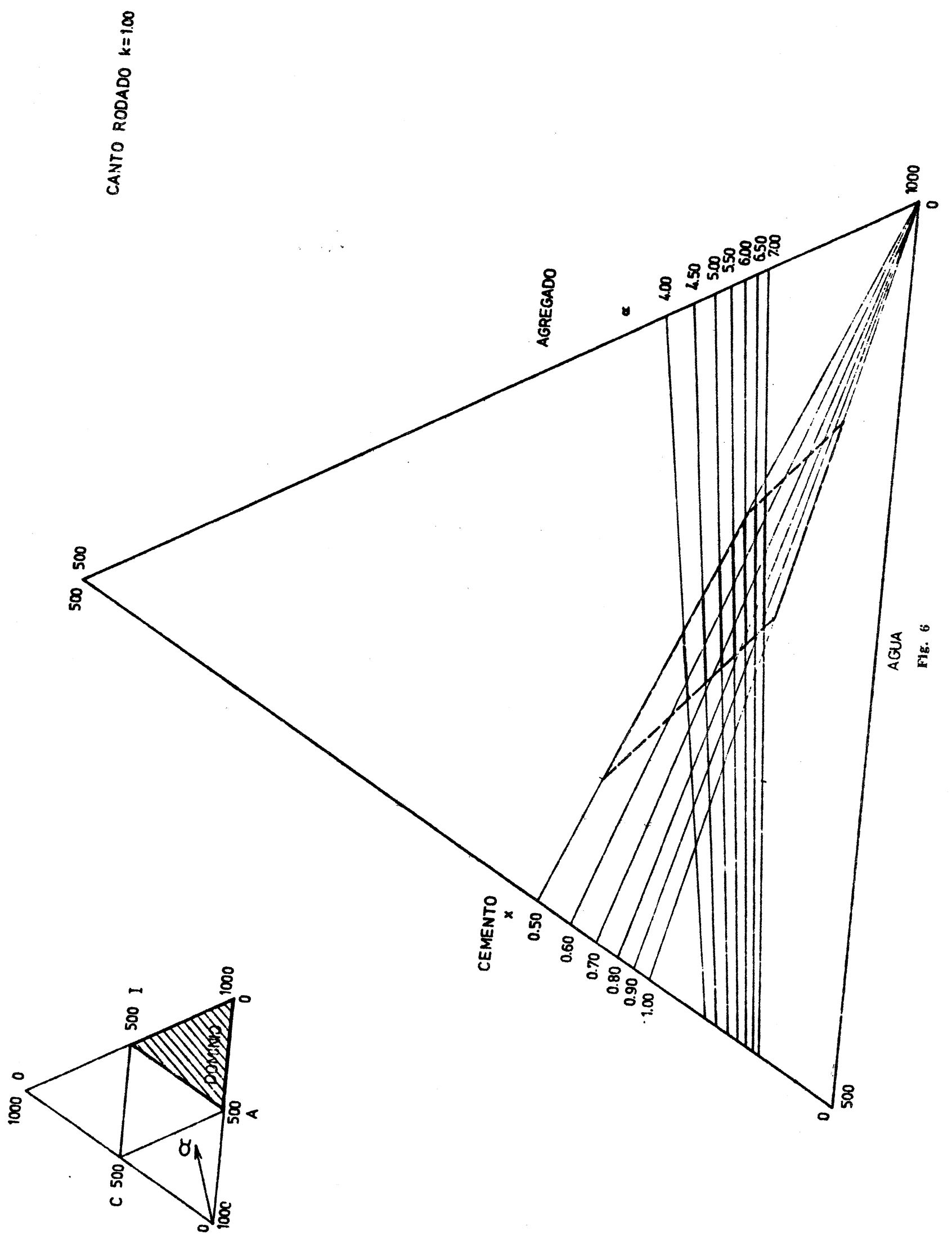




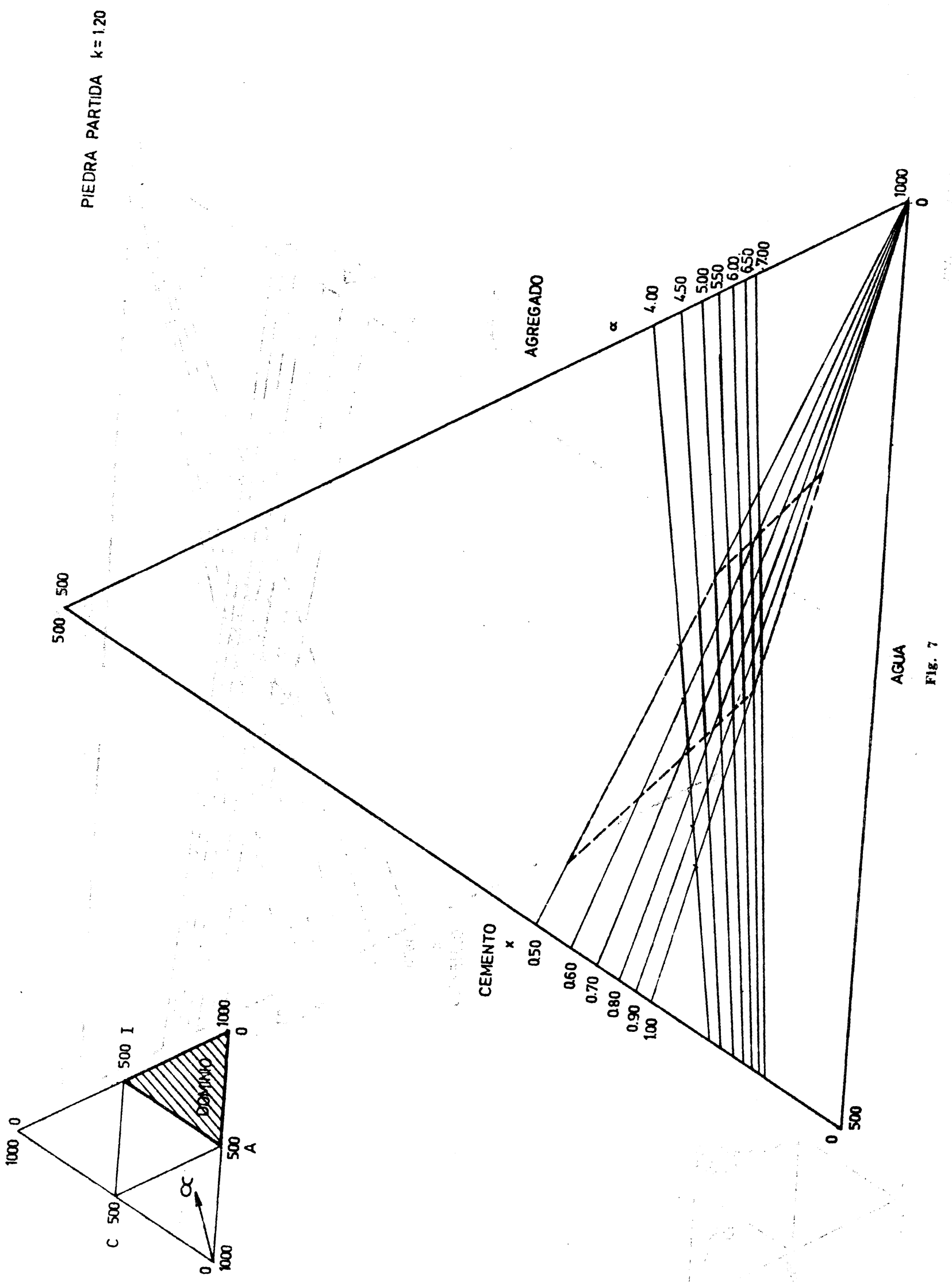

22 


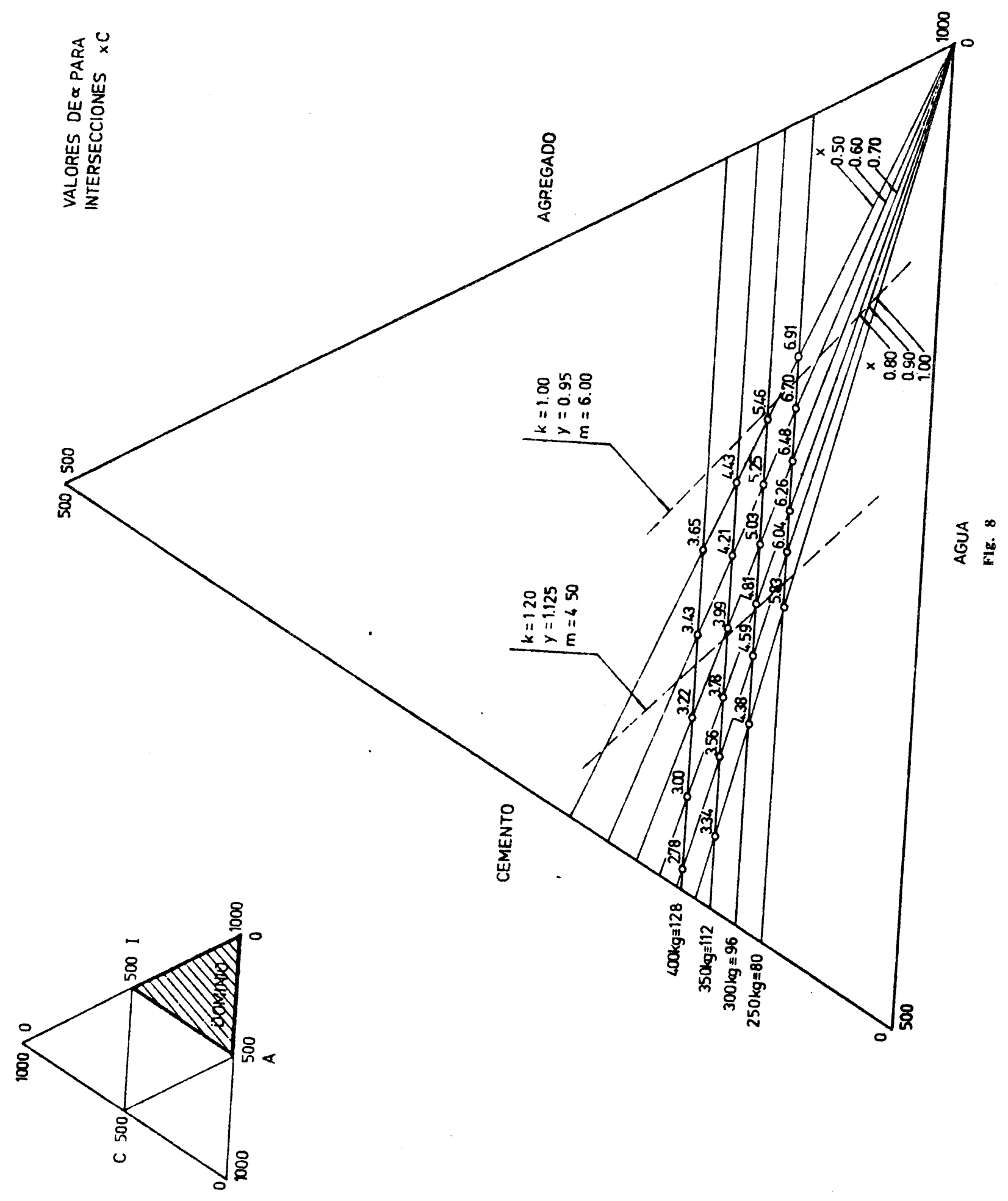




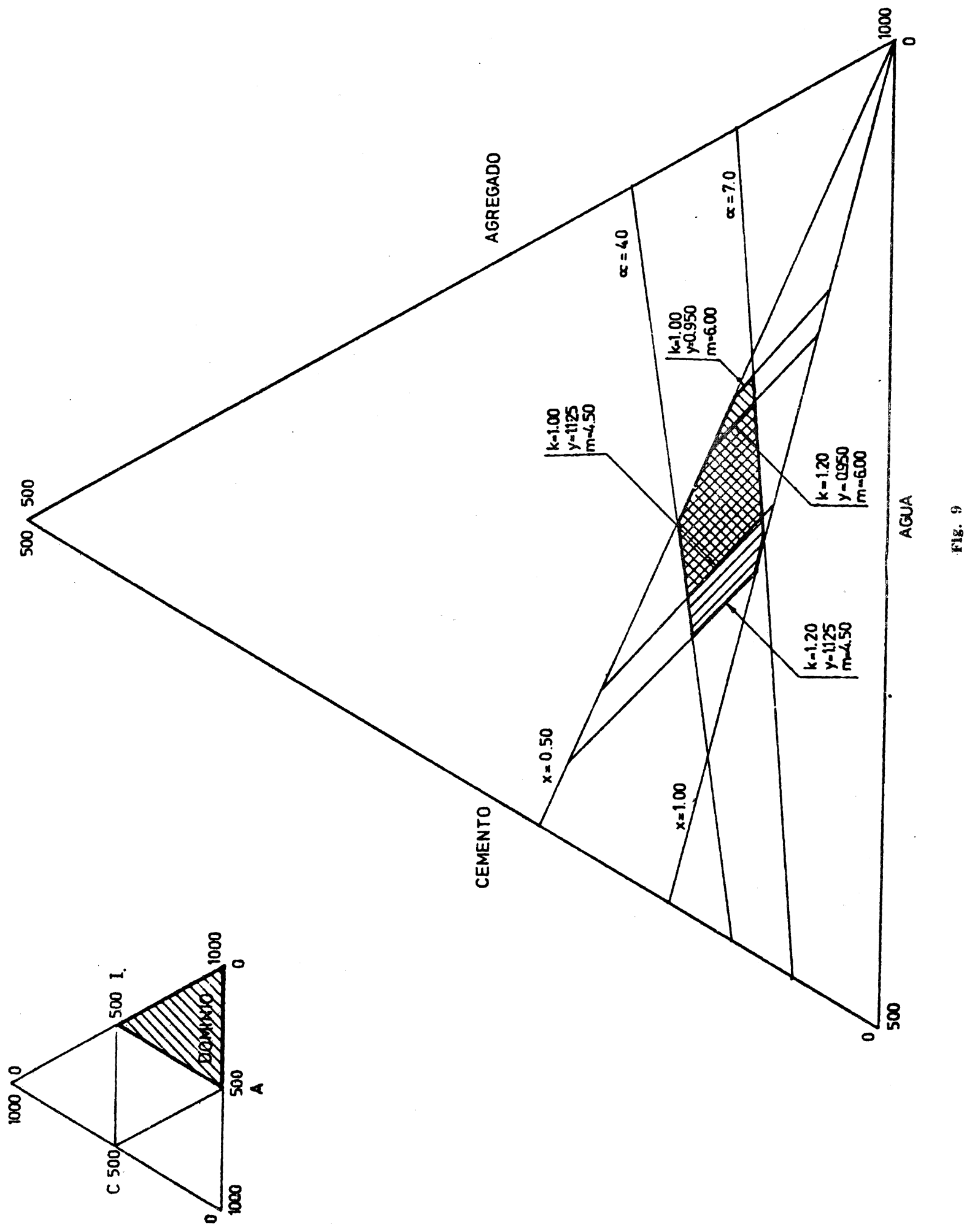




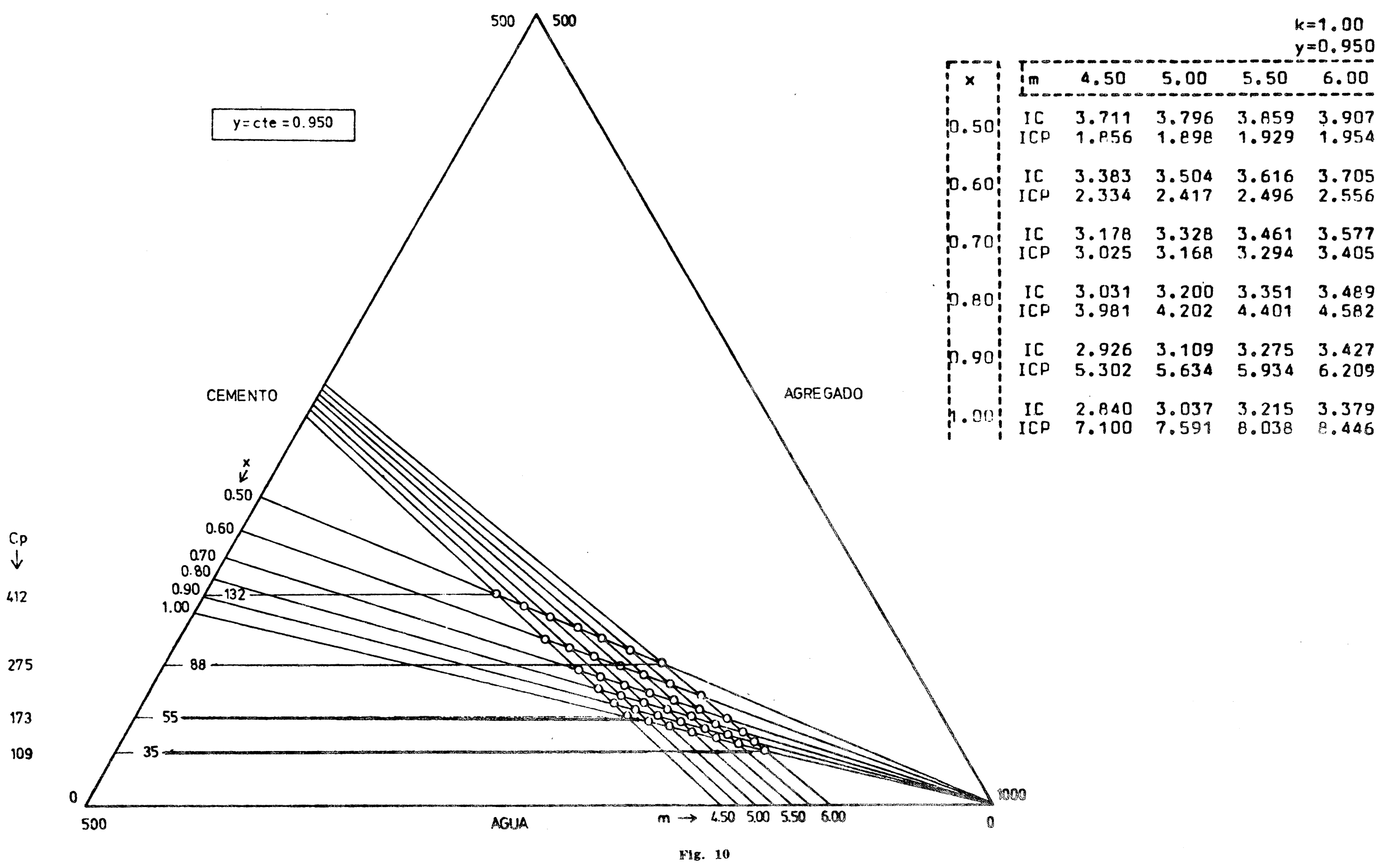




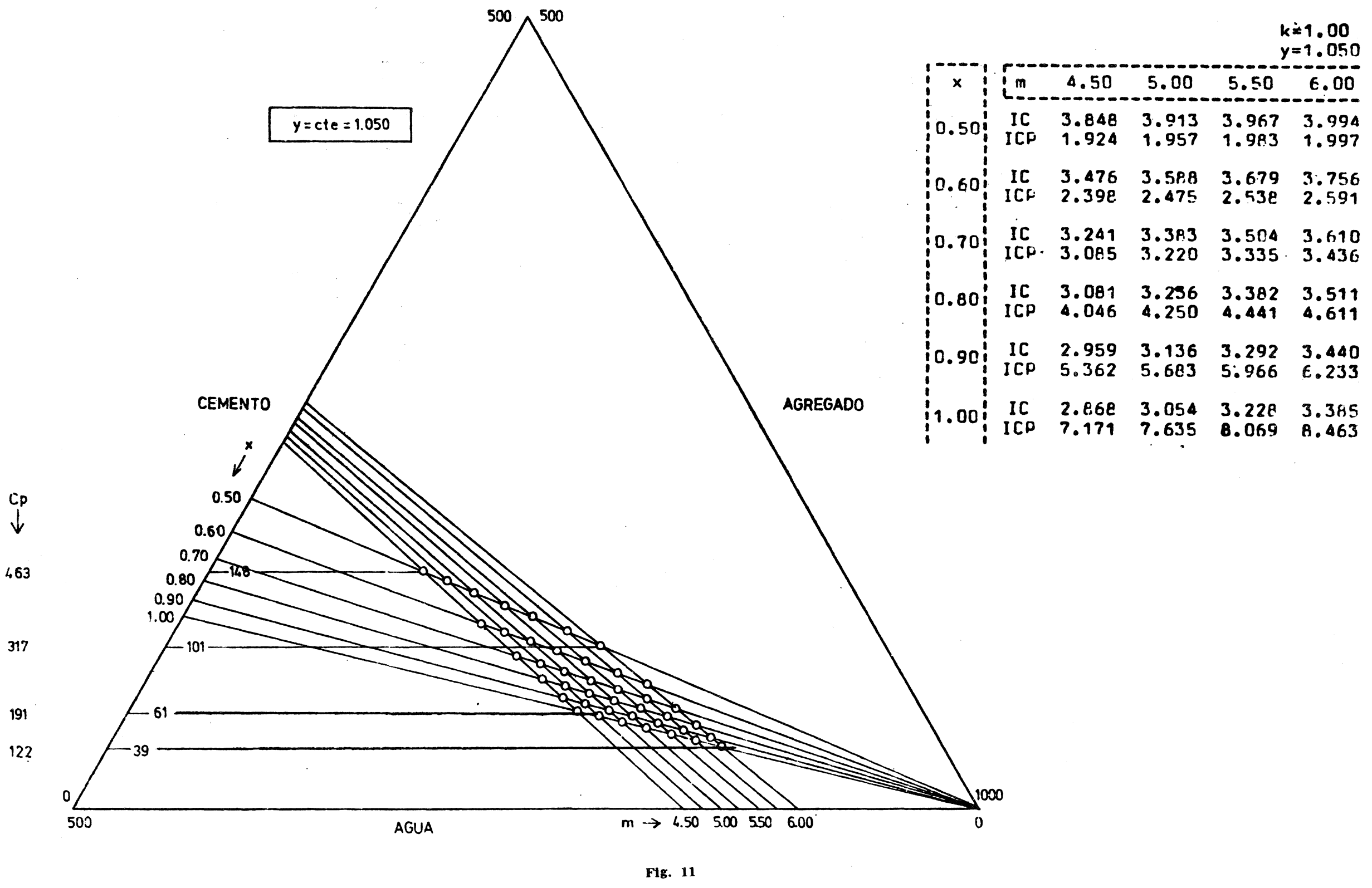




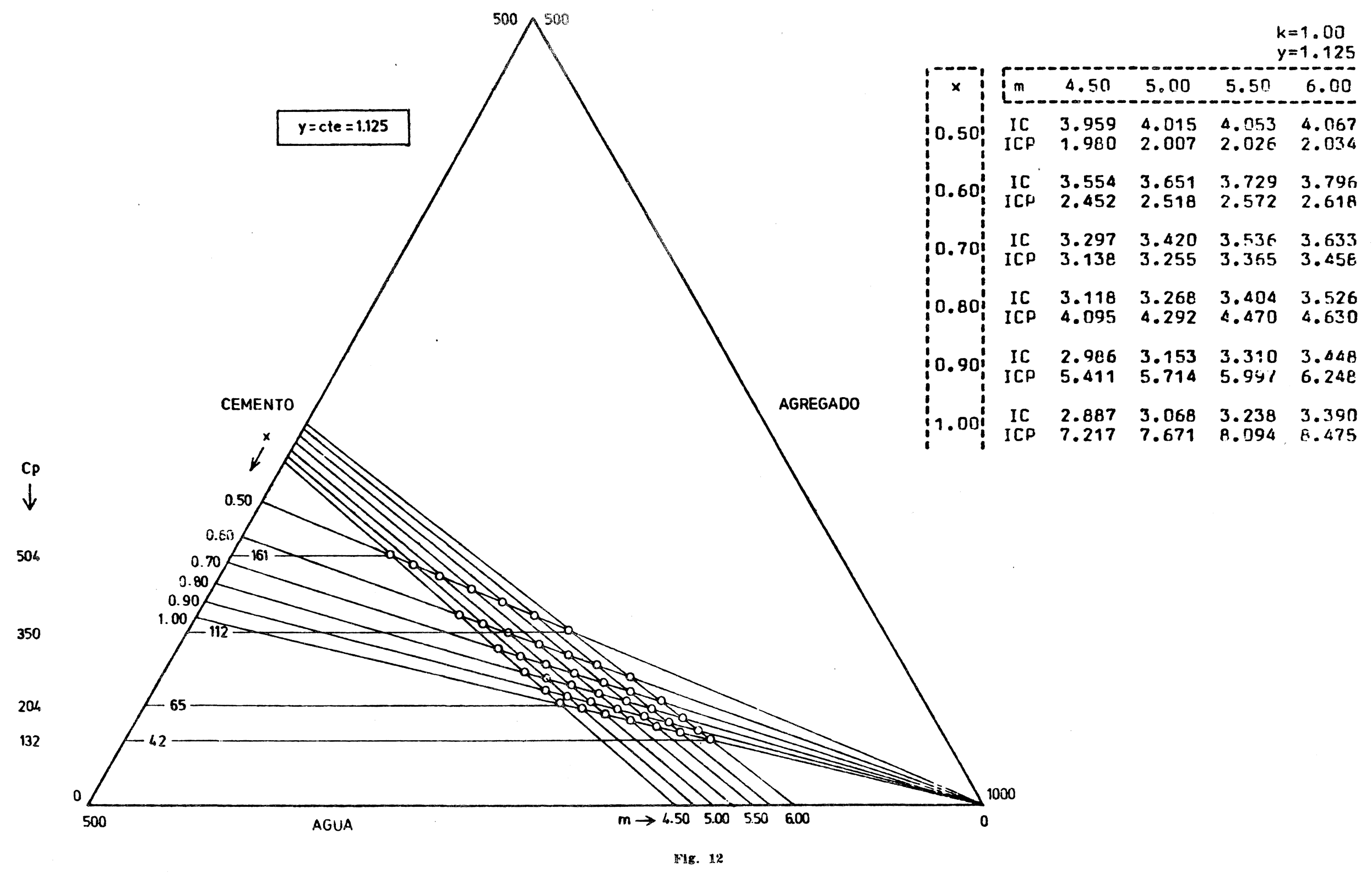




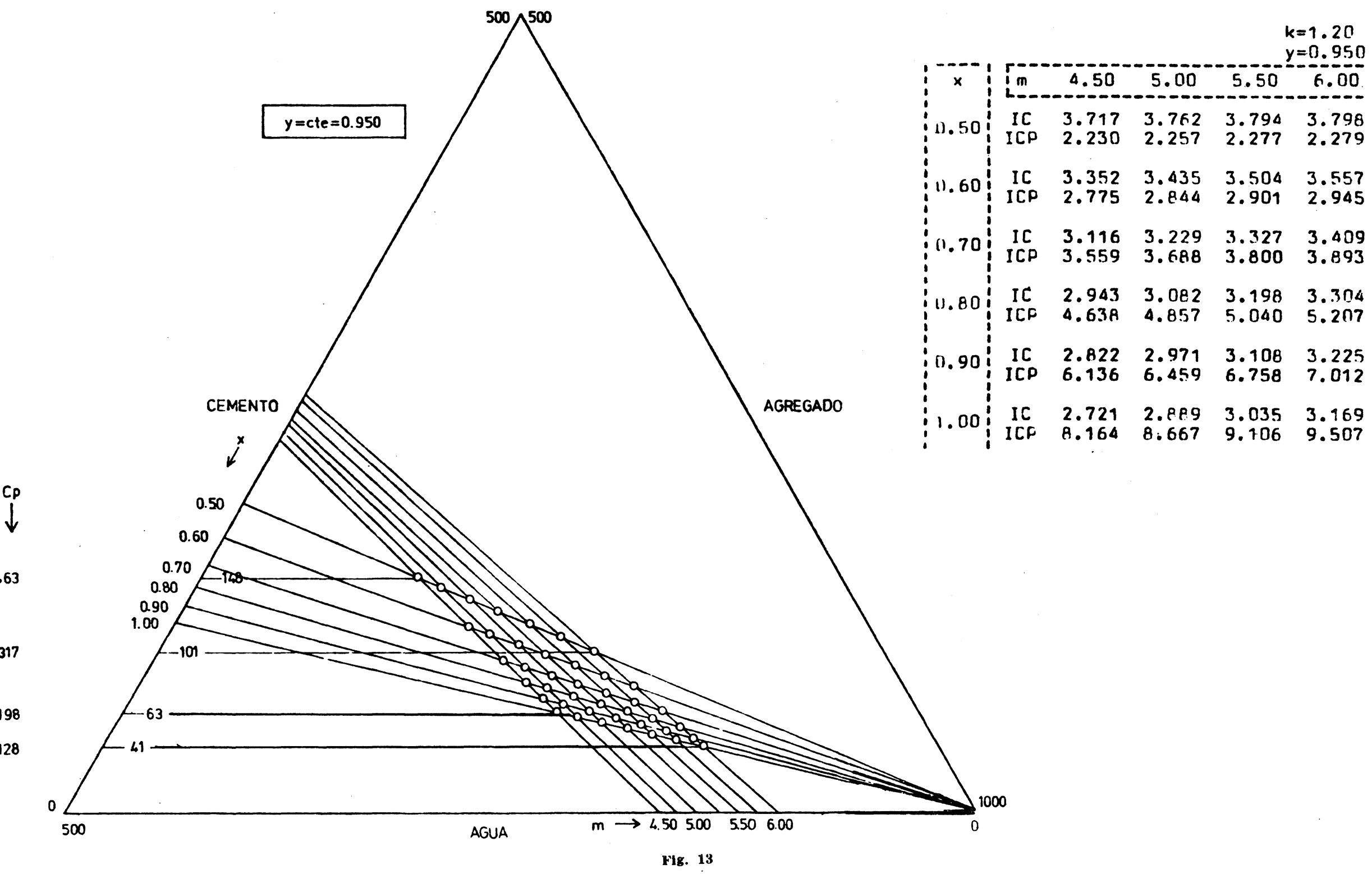




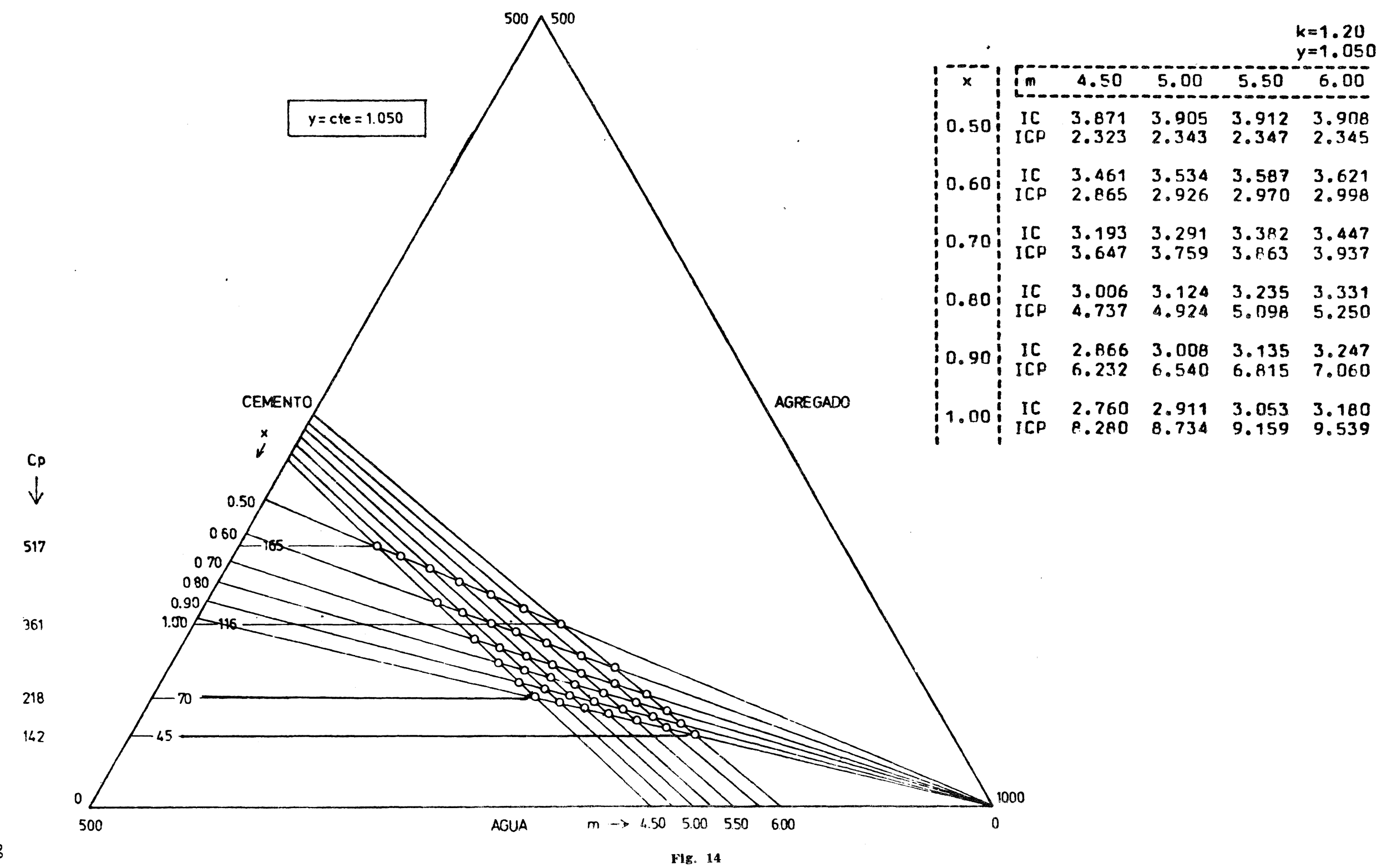




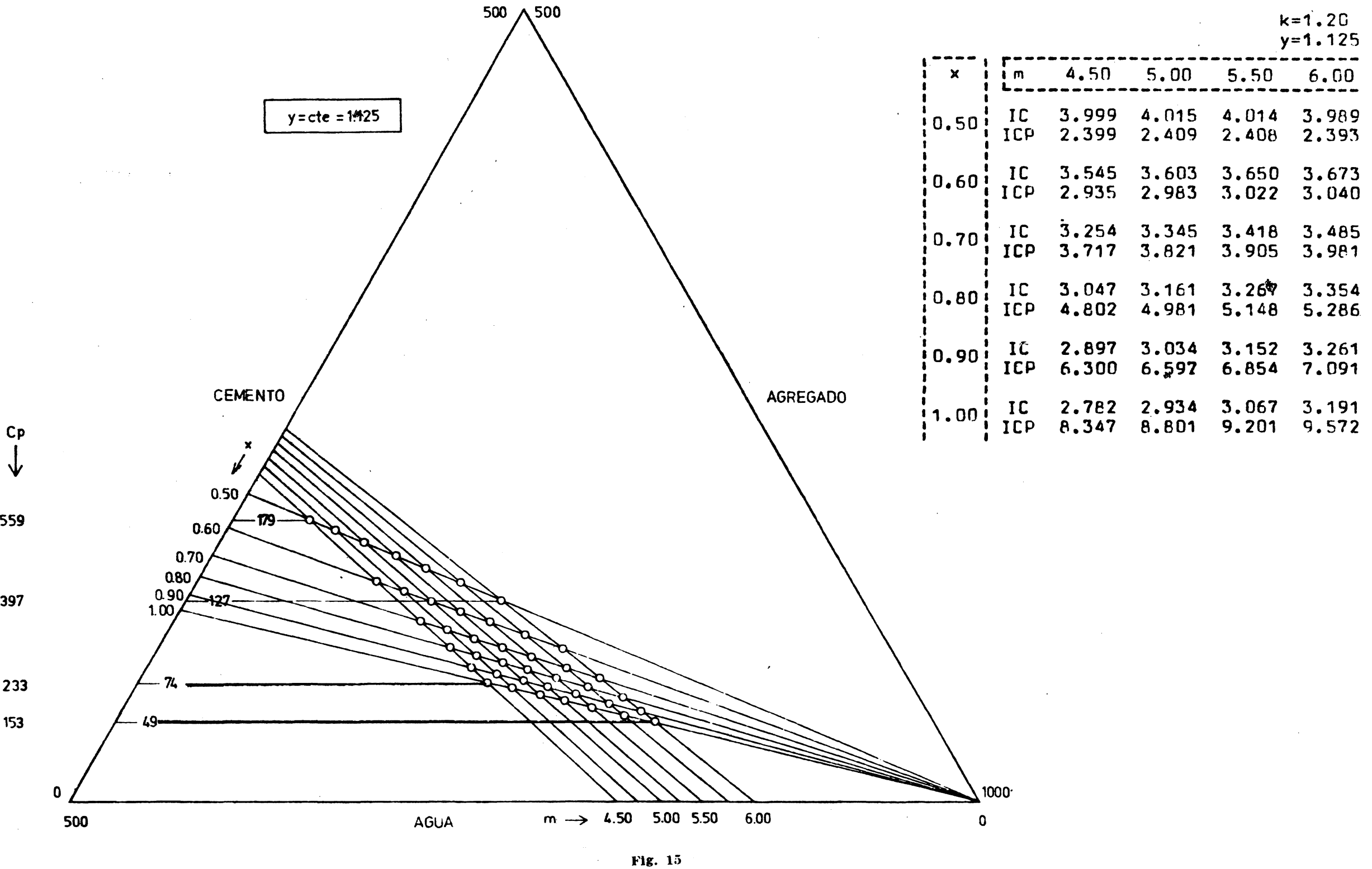




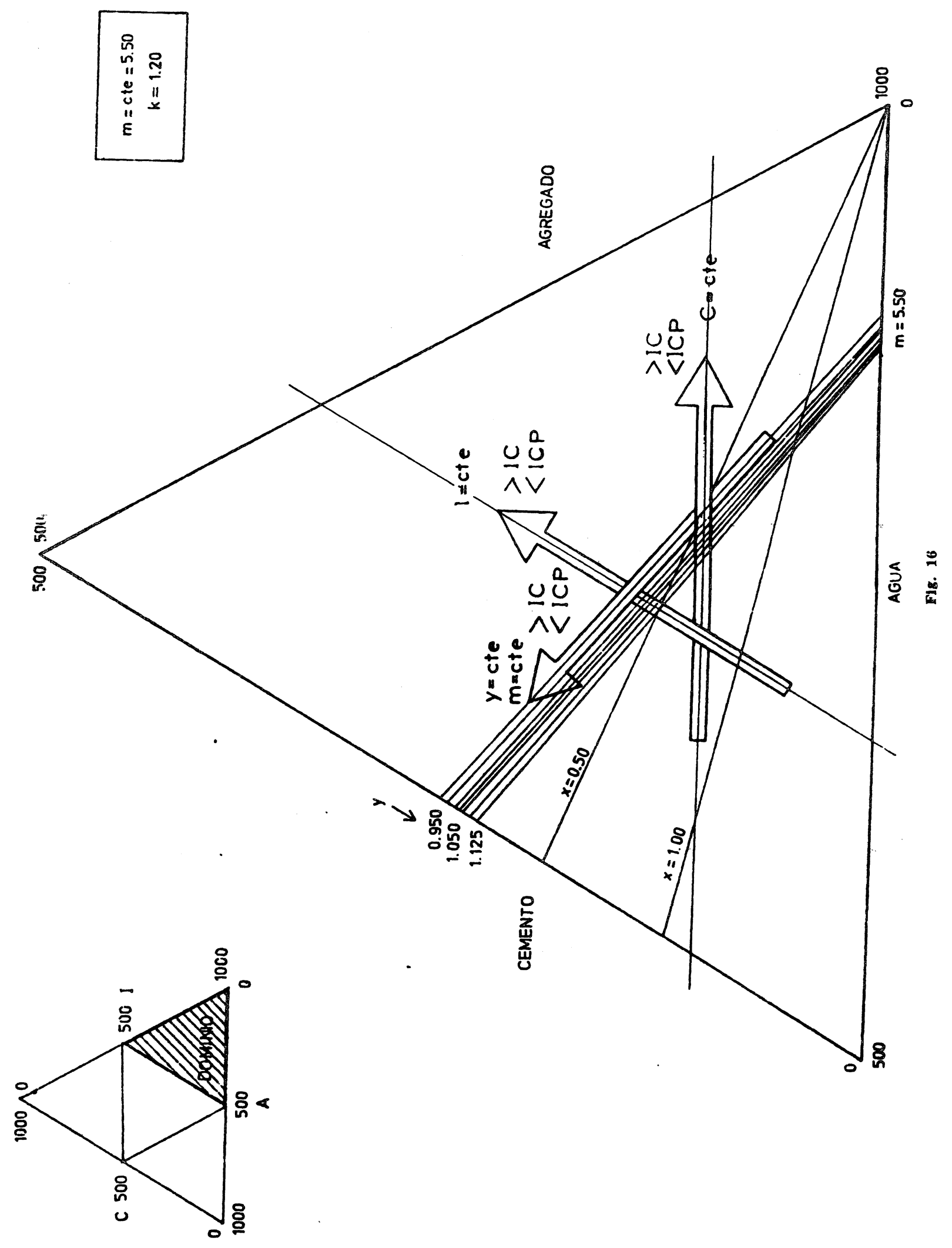




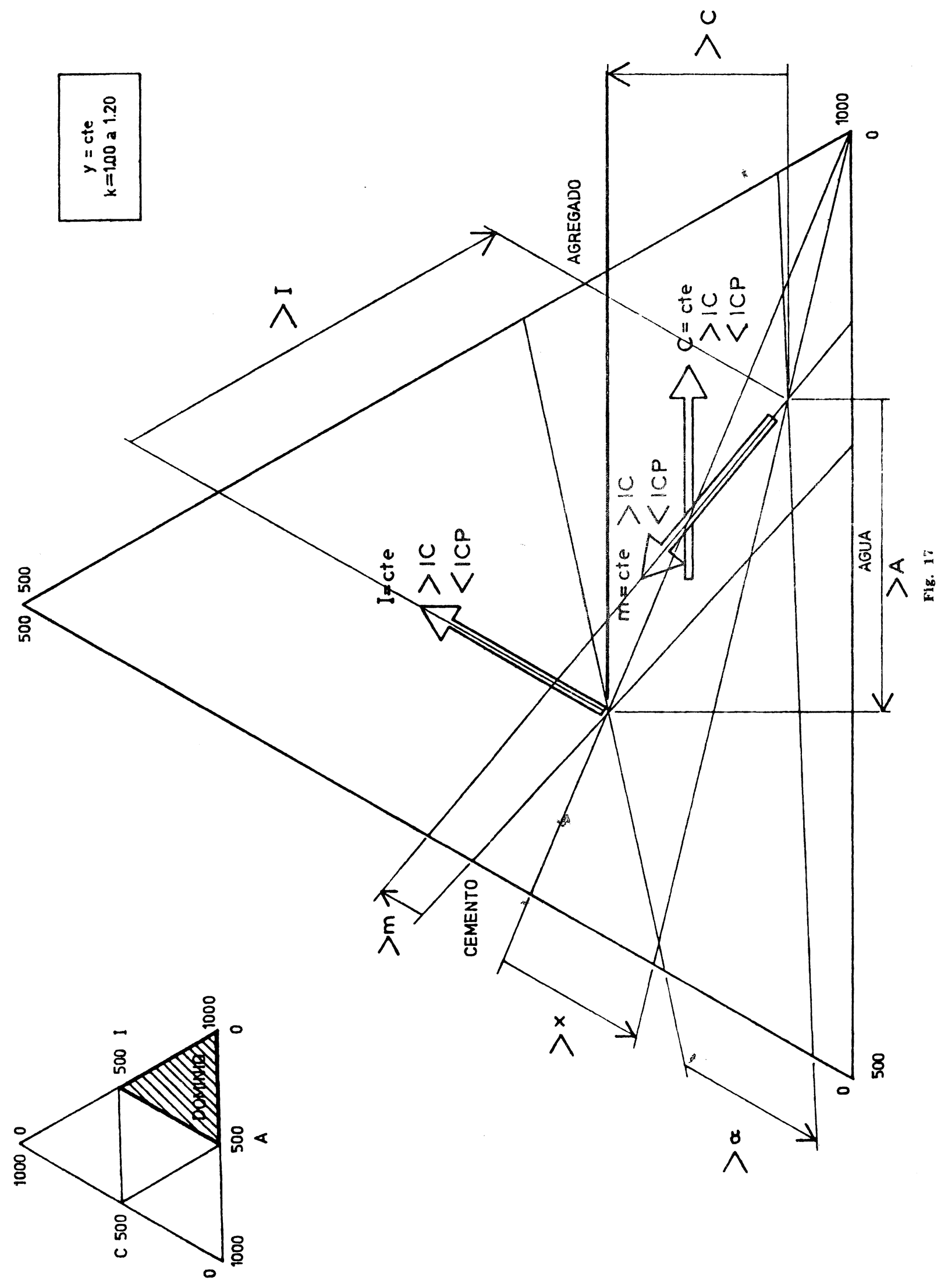




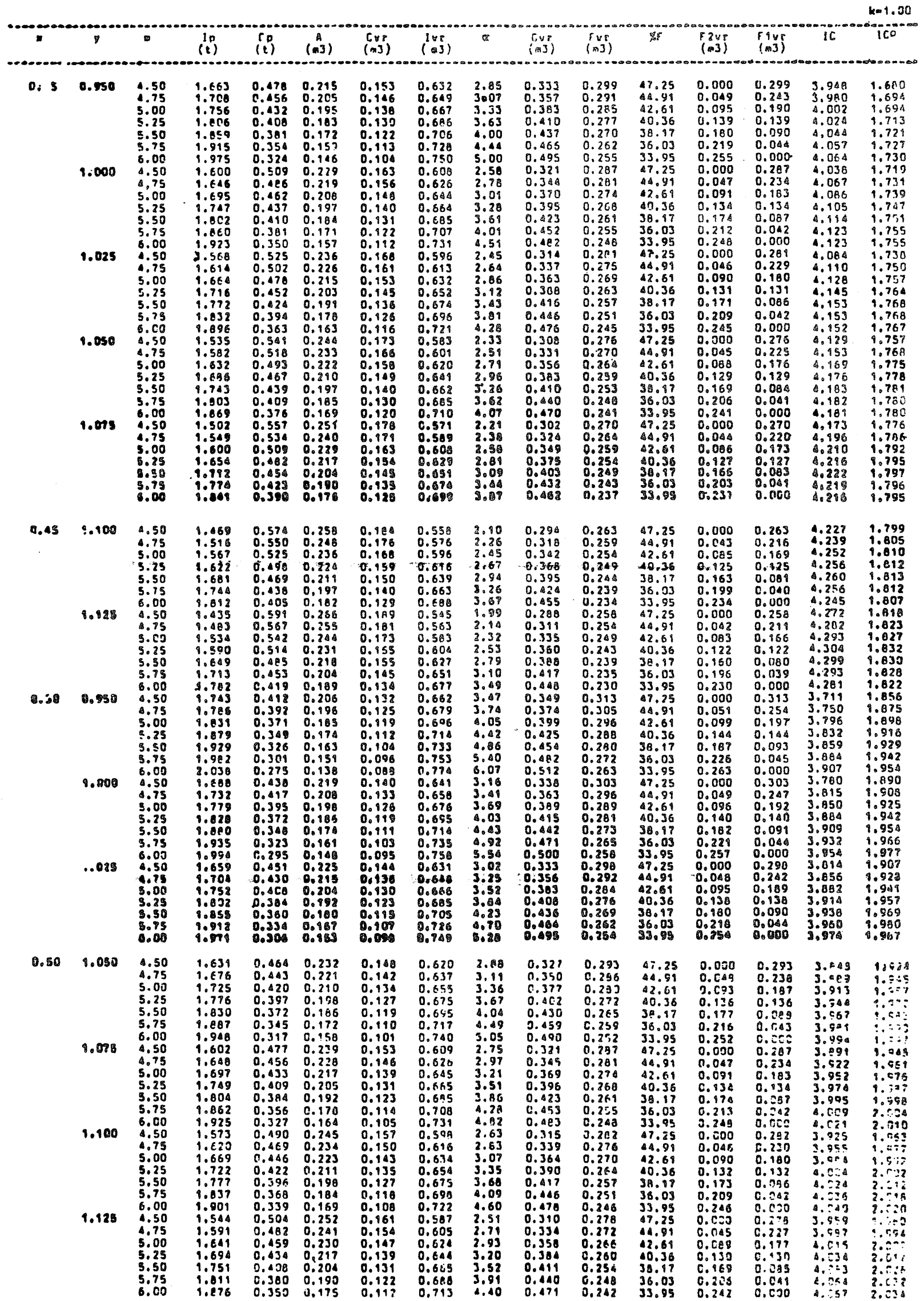

Fig. 18.-Dosificaciones procesadas por computación. 


\begin{tabular}{|c|c|c|c|c|c|c|c|c|c|c|c|c|c|c|c|}
\hline$\cdots$ & RE SU & & (b) & $\begin{array}{l}c p \\
(t)\end{array}$ & $\left(\operatorname{an}^{n}\right)$ & $\begin{array}{l}\text { Cur } \\
(-3)\end{array}$ & $\left(\begin{array}{l}\ln 8 \\
(\rightarrow 3)\end{array}\right.$ & a & $\begin{array}{l}\text { Gur' } \\
\left.(\mathrm{m})^{\circ}\right)\end{array}$ & $\left(\begin{array}{l}\operatorname{cr} \\
(\mathrm{m})\end{array}\right)$ & 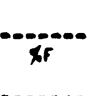 & 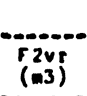 & $\begin{array}{l}\text { Fivr } \\
\text { (m3) }\end{array}$ & IC & k=1.00 \\
\hline$\ldots .45$ & $\begin{array}{l}0.950 \\
1.000 \\
1.025 \\
1.050 \\
1.075 \\
1.100 \\
1.125 \\
9.950 \\
1.000 \\
1.025 \\
1.050 \\
1.075 \\
1.100 \\
1.125 \\
0.950 \\
1.000 \\
1.025 \\
1.050 \\
1.075 \\
1.100 \\
1.125 \\
0.950 \\
1.000 \\
1.025 \\
1.050 \\
1.075 \\
9.100 \\
1.125 \\
0.950 \\
1.000 \\
1.025 \\
9.050 \\
1.075 \\
1.100 \\
1.123\end{array}$ & $\begin{array}{l}5.50 \\
5.50 \\
5.50 \\
5.50 \\
5.50 \\
5.50 \\
5.50 \\
5.30 \\
5.50 \\
5.50 \\
5.50 \\
5.50 \\
5.50 \\
5.50 \\
5.50 \\
5.50 \\
5.50 \\
150 \\
5.50 \\
5.50 \\
5.50 \\
5.80 \\
5.50 \\
5.50 \\
3.50 \\
550 \\
5.50 \\
5.50 \\
5.50 \\
5.30 \\
5.50 \\
5.50 \\
5.50 \\
3.50 \\
3.50\end{array}$ & 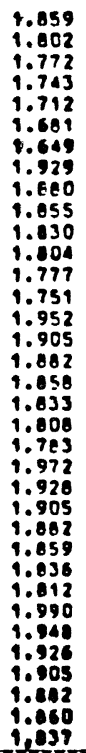 & $\begin{array}{l}0.381 \\
0.410 \\
0.424 \\
0.439 \\
0.454 \\
0.469 \\
0.485 \\
0.326 \\
0.340 \\
0.360 \\
0.372 \\
0.304 \\
0.396 \\
0.406 \\
0.308 \\
0.329 \\
0.339 \\
0.350 \\
0.361 \\
0.372 \\
0.384 \\
0.291 \\
0.311 \\
0.321 \\
0.331 \\
0.341 \\
0.352 \\
0.362 \\
0.217 \\
0.293 \\
0.304 \\
0.314 \\
0.324 \\
0.333 \\
0.343\end{array}$ & $\begin{array}{l}0.112 \\
0.184 \\
0.191 \\
0.197 \\
0.204 \\
0.211 \\
0.218 \\
0.163 \\
0.111 \\
0.190 \\
0.188 \\
0.192 \\
0.198 \\
0.204 \\
0.160 \\
0.171 \\
0.176 \\
0.182 \\
0.190 \\
0.194 \\
0.200 \\
0.157 \\
0.160 \\
0.173 \\
0.170 \\
0.184 \\
0.190 \\
0.196 \\
0.195 \\
0.165 \\
0.171 \\
0.176 \\
0.191 \\
0.197 \\
0.198\end{array}$ & $\begin{array}{l}0.122 \\
0.131 \\
0.136 \\
0.110 \\
0.145 \\
0.150 \\
0.155 \\
0.104 \\
0.111 \\
0.115 \\
0.119 \\
0.123 \\
0.127 \\
0.131 \\
0.098 \\
0.105 \\
0.109 \\
0.112 \\
0.116 \\
0.119 \\
0.123 \\
0.093 \\
0.100 \\
0.103 \\
0.106 \\
0.109 \\
0.113 \\
0.116 \\
0.009 \\
0.095 \\
0.097 \\
0.100 \\
0.104 \\
0.107 \\
0.810\end{array}$ & $\begin{array}{l}0.706 \\
0.685 \\
0.674 \\
0.762 \\
0.651 \\
0.639 \\
0.627 \\
0.733 \\
0.714 \\
0.705 \\
0.695 \\
0.685 \\
0.675 \\
0.665 \\
0.742 \\
0.724 \\
0.715 \\
0.706 \\
0.697 \\
0.687 \\
0.678 \\
0.7199 \\
0.733 \\
0.724 \\
0.795 \\
0.706 \\
0.697 \\
0.600 \\
0.756 \\
0.740 \\
0.732 \\
0.724 \\
0.715 \\
8.707 \\
0.690\end{array}$ & $\begin{array}{l}4.00 \\
3.61 \\
3.43 \\
3.26 \\
3.09 \\
2.94 \\
2.79 \\
4.86 \\
4.43 \\
4.23 \\
4.04 \\
3.86 \\
3.60 \\
3.52 \\
5.20 \\
4.76 \\
4.55 \\
4.35 \\
4.16 \\
3.90 \\
3.81 \\
5.55 \\
5.00 \\
4.07 \\
4.66 \\
4.47 \\
4.28 \\
4.10 \\
5.90 \\
5.41 \\
5.19 \\
4.97 \\
4.77 \\
4.58 \\
4.39\end{array}$ & $\begin{array}{l}0.437 \\
0.423 \\
0.416 \\
0.410 \\
0.403 \\
0.395 \\
0.388 \\
0.454 \\
0.442 \\
0.436 \\
0.430 \\
0.423 \\
0.417 \\
0.419 \\
0.459 \\
0.451 \\
0.441 \\
0.4137 \\
0.430 \\
0.425 \\
0.419 \\
0.464 \\
0.452 \\
0.447 \\
0.442 \\
0.437 \\
0.443 \\
0.426 \\
0.467 \\
0.457 \\
0.454 \\
0.448 \\
0.441 \\
0.4436 \\
0.431\end{array}$ & $\begin{array}{l}0.270 \\
0.261 \\
0.257 \\
0.253 \\
0.249 \\
0.244 \\
0.239 \\
0.280 \\
0.273 \\
0.269 \\
0.265 \\
0.261 \\
0.257 \\
0.254 \\
0.284 \\
0.271 \\
0.272 \\
0.269 \\
0.265 \\
0.262 \\
0.258 \\
0.286 \\
0.279 \\
0.276 \\
0.373 \\
0.270 \\
0.266 \\
0.263 \\
0.216 \\
0.282 \\
0.2140 \\
0.279 \\
0.273 \\
0.269 \\
0.266\end{array}$ & 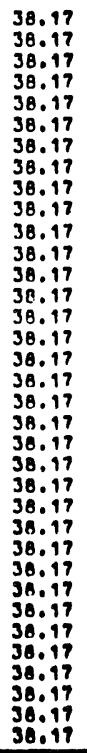 & $\begin{array}{l}0.180 \\
0.174 \\
0.171 \\
0.169 \\
0.166 \\
0.163 \\
0.160 \\
0.187 \\
0.182 \\
0.180 \\
0.177 \\
0.174 \\
0.173 \\
0.169 \\
0.189 \\
0.186 \\
0.182 \\
0.180 \\
0.177 \\
0.175 \\
0.172 \\
0.191 \\
0.186 \\
0.184 \\
0.182 \\
0.180 \\
0.177 \\
0.173 \\
0.192 \\
0.180 \\
0.187 \\
0.185 \\
0.182 \\
0.180 \\
0.170\end{array}$ & $\begin{array}{l}0.090 \\
0.087 \\
0.086 \\
0.084 \\
0.083 \\
0.081 \\
0.080 \\
0.093 \\
0.091 \\
0.090 \\
0.088 \\
0.087 \\
0.086 \\
0.085 \\
0.094 \\
0.093 \\
0.099 \\
0.090 \\
0.088 \\
0.087 \\
0.086 \\
0.095 \\
0.093 \\
0.092 \\
0.091 \\
0.090 \\
0.089 \\
0.088 \\
0.096 \\
0.094 \\
0.093 \\
0.093 \\
0.099 \\
0.090 \\
0.089\end{array}$ & $\begin{array}{l}4.044 \\
4.114 \\
4.153 \\
4.183 \\
4.222 \\
4.260 \\
4.299 \\
3.859 \\
3.909 \\
3.936 \\
3.967 \\
3.995 \\
4.024 \\
4.053 \\
3.797 \\
3.846 \\
3.874 \\
3.895 \\
3.923 \\
3.944 \\
3.972 \\
3.744 \\
3.792 \\
3.813 \\
3.833 \\
3.054 \\
3.881 \\
3.901 \\
3.700 \\
3.740 \\
3.754 \\
3.773 \\
3.800 \\
3.820 \\
3.840\end{array}$ & $\begin{array}{l}1.721 \\
1.751 \\
9.768 \\
1.789 \\
1.797 \\
1.811 \\
1.830 \\
1.929 \\
1.954 \\
1.969 \\
1.983 \\
1.998 \\
2.012 \\
2.026 \\
2.025 \\
2.051 \\
2.066 \\
2.077 \\
2.092 \\
2.103 \\
2.118 \\
2.129 \\
2.157 \\
2.168 \\
2.180 \\
2.192 \\
2.207 \\
2.219 \\
2.244 \\
2.269 \\
2.279 \\
2.289 \\
2.305 \\
2.319 \\
2.329\end{array}$ \\
\hline$x$ & $y$ & $m$ & $\begin{array}{l}1 p \\
(t)\end{array}$ & $\begin{array}{l}c p \\
(t)\end{array}$ & $(\hat{m} 3)$ & $\begin{array}{l}\text { Cvr } \\
(\mathrm{mJ})\end{array}$ & 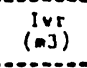 & $\alpha$ & $\begin{array}{l}\text { cur } \\
(m 3)\end{array}$ & $\begin{array}{l}\text { (ver } \\
(m 3)\end{array}$ & a & $\begin{array}{l}\text { ive } \\
(-3)\end{array}$ & $\begin{array}{l}r \text { ivr } \\
(-3)\end{array}$ & is. & 160 \\
\hline 0.58 & $\begin{array}{l}0.950 \\
1.000 \\
1.025 \\
1.050 \\
1.075 \\
1.100 \\
1.125 \\
0.950 \\
1.000 \\
1.025 \\
1.050 \\
1.075 \\
1.100 \\
1.125 \\
0.950 \\
1.000 \\
1.025 \\
1.050 \\
1.075 \\
1.100 \\
1.125 \\
0.950 \\
1.000 \\
1.025 \\
1.050 \\
1.075 \\
1.100 \\
1.125 \\
0.950 \\
1.000 \\
1.025 \\
1.050 \\
1.075 \\
1.100 \\
1.125\end{array}$ & 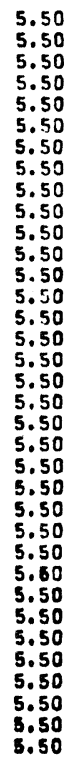 & $\begin{array}{l}2.007 \\
1.966 \\
1.946 \\
1.925 \\
1.903 \\
1.882 \\
1.860 \\
2.022 \\
1.983 \\
1.963 \\
1.943 \\
1.922 \\
1.902 \\
1.881 \\
2.054 \\
2.098 \\
2.000 \\
1.982 \\
1.963 \\
1.944 \\
1.925 \\
2.081 \\
2.047 \\
2.030 \\
2.013 \\
1.9996 \\
1.978 \\
1.961 \\
2.121 \\
2.0991 \\
2.076 \\
2.061 \\
2.043 \\
2.030 \\
2.0918\end{array}$ & $\begin{array}{l}0.254 \\
0.281 \\
0.290 \\
0.298 \\
0.307 \\
0.317 \\
0.326 \\
0.252 \\
0.268 \\
0.276 \\
0.285 \\
0.293 \\
0.301 \\
0.310 \\
0.226 \\
0.240 \\
0.247 \\
0.255 \\
0.262 \\
0.269 \\
0.277 \\
0.205 \\
0.218 \\
0.224 \\
0.230 \\
0.239 \\
0.243 \\
0.250 \\
0.173 \\
0.183 \\
0.189 \\
0.194 \\
0.199 \\
0.204 \\
0.209\end{array}$ & $\begin{array}{l}0.153 \\
0.153 \\
0.168 \\
0.173 \\
0.178 \\
0.184 \\
0.189 \\
0.151 \\
0.161 \\
0.166 \\
0.1711 \\
0.176 \\
0.181 \\
0.186 \\
0.1197 \\
0.156 \\
0.161 \\
0.166 \\
0.170 \\
0.175 \\
0.180 \\
0.141 \\
0.152 \\
0.157 \\
0.161 \\
0.166 \\
0.190 \\
0.175 \\
0.139 \\
0.147 \\
0.151 \\
0.155 \\
0.159 \\
0.163 \\
0.167\end{array}$ & $\begin{array}{l}0.084 \\
0.090 \\
0.093 \\
0.096 \\
0.098 \\
0.101 \\
0.104 \\
0.0811 \\
0.086 \\
0.088 \\
0.091 \\
0.055 \\
0.096 \\
0.099 \\
0.072 \\
0.077 \\
0.079 \\
0.081 \\
0.084 \\
0.086 \\
0.089 \\
0.066 \\
0.070 \\
0.077 \\
0.074 \\
0.076 \\
0.078 \\
0.000 \\
0.055 \\
0.059 \\
0.060 \\
0.062 \\
0.064 \\
0.065 \\
0.067\end{array}$ & $\begin{array}{l}0.763 \\
0.747 \\
0.739 \\
0.731 \\
0.723 \\
0.715 \\
0.7707 \\
0.7768 \\
0.753 \\
0.746 \\
0.738 \\
0.7731 \\
0.723 \\
0.775 \\
0.781 \\
0.767 \\
0.760 \\
0.753 \\
0.746 \\
0.739 \\
0.732 \\
0.791 \\
0.778 \\
0.777 \\
0.765 \\
0.758 \\
0.752 \\
0.745 \\
0.806 \\
0.795 \\
0.789 \\
0.783 \\
0.777 \\
0.771 \\
0.766\end{array}$ & $\begin{array}{r}6.24 \\
5.74 \\
5.51 \\
5.29 \\
5.00 \\
4.98 \\
4.68 \\
6.59 \\
6.07 \\
5.83 \\
5.60 \\
5.38 \\
5.17 \\
4.97 \\
7.45 \\
6.89 \\
6.53 \\
6.38 \\
6.94 \\
5.92 \\
5.70 \\
8.31 \\
7.71 \\
7.49 \\
7.16 \\
6.91 \\
6.66 \\
6.43 \\
10.04 \\
9.35 \\
9.03 \\
8.72 \\
8.43 \\
8.16 \\
7.89\end{array}$ & $\begin{array}{l}0.472 \\
0.46,2 \\
0.457 \\
0.451 \\
0.448 \\
0.413 \\
0.438 \\
0.474 \\
0.466 \\
0.462 \\
0.457 \\
0.450 \\
0.448 \\
0.442 \\
0.434 \\
0.474 \\
0.470 \\
0.467 \\
0.469 \\
0.457 \\
0.452 \\
0.489 \\
0.489 \\
0.4777 \\
0.473 \\
0.449 \\
0.445 \\
0.461 \\
0.450 \\
0.499 \\
0.489 \\
0.484 \\
0.480 \\
0.479 \\
0.474\end{array}$ & $\begin{array}{l}0.292 \\
0.285 \\
0.272 \\
0.279 \\
0.276 \\
0.273 \\
0.270 \\
0.293 \\
0.287 \\
0.285 \\
0.292 \\
0.278 \\
0.276 \\
0.273 \\
0.298 \\
0.293 \\
0.290 \\
0.288 \\
0.285 \\
0.282 \\
0.279 \\
0.302 \\
0.296 \\
0.291 \\
0.292 \\
0.219 \\
0.289 \\
0.283 \\
0.307 \\
0.303 \\
0.302 \\
0.299 \\
0.296 \\
0.295 \\
0.292\end{array}$ & $\begin{array}{l}38.17 \\
38.97 \\
38.17 \\
38.17 \\
39.17 \\
33.17 \\
36.197 \\
38.17 \\
34.17 \\
38.17 \\
34.17 \\
30.17 \\
36.17 \\
38.17 \\
38.17 \\
38.17 \\
38.17 \\
38.17 \\
38.17 \\
38.17 \\
38.17 \\
34.17 \\
38.17 \\
38.17 \\
39.17 \\
38.17 \\
38.17 \\
30.17 \\
38.17 \\
38.17 \\
38.17 \\
38.17 \\
38.97 \\
38.17 \\
38.17\end{array}$ & 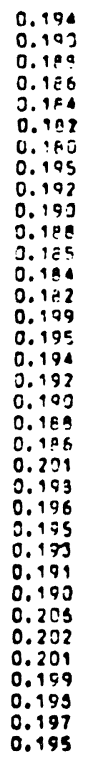 & 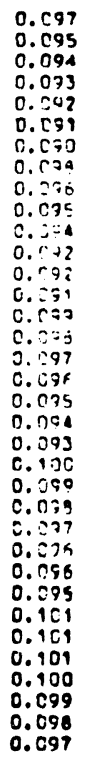 & 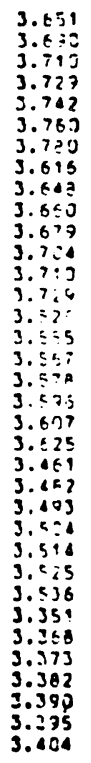 & 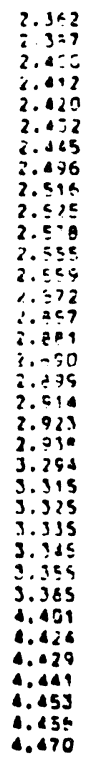 \\
\hline$n$ & $y$ & & $(t p)$ & $(t)$ & $\left(\begin{array}{l}n \\
m\end{array}\right)$ & (m3) & $\left(\begin{array}{l}\ln 5 \\
(m 3)\end{array}\right.$ & $\alpha$ & $\begin{array}{l}\text { Cup } \\
\text { (nJ) }\end{array}$ & $\begin{array}{l}\text { (ve } \\
(m 3)\end{array}$ & 85 & $\begin{array}{l}2 \times 5 \\
(-3)\end{array}$ & $\begin{array}{l}(1 v 8 \\
(-3)\end{array}$ & is & : \\
\hline 0.90 & $\begin{array}{l}0.950 \\
1.000 \\
1.025 \\
1.050 \\
1.075 \\
1.100 \\
1.125 \\
0.950 \\
1.000 \\
1.025 \\
1.050 \\
1.075 \\
1.100 \\
1.125\end{array}$ & $\begin{array}{l}5.50 \\
5.50 \\
5.50 \\
5.50 \\
5.50 \\
5.50 \\
5.50 \\
5.50 \\
5.50 \\
5.50 \\
5.50 \\
3.50 \\
5.50 \\
\mathbf{5 . 5 0}\end{array}$ & $\begin{array}{l}2.150 \\
2.123 \\
2.109 \\
2.095 \\
2.081 \\
2.067 \\
2.053 \\
2.173 \\
2.147 \\
2.134 \\
2.122 \\
2.109 \\
2.096 \\
2.083\end{array}$ & $\begin{array}{l}0.150 \\
0.158 \\
0.163 \\
0.167 \\
0.179 \\
0.176 \\
0.180 \\
0.132 \\
0.139 \\
0.143 \\
0.1417 \\
0.151 \\
0.154 \\
0.158\end{array}$ & $\begin{array}{l}0.135 \\
0.1143 \\
0.146 \\
0.150 \\
0.154 \\
0.158 \\
0.162 \\
0.132 \\
0.139 \\
0.143 \\
0.147 \\
0.1191 \\
0.154 \\
0.158\end{array}$ & $\begin{array}{l}0.048 \\
0.051 \\
0.052 \\
0.053 \\
0.053 \\
0.056 \\
0.050 \\
0.042 \\
0.045 \\
0.046 \\
0.047 \\
0.048 \\
0.044 \\
0.051\end{array}$ & $\begin{array}{l}0.817 \\
0.807 \\
0.801 \\
0.796 \\
0.791 \\
0.786 \\
0.780 \\
0.826 \\
0.816 \\
0.819 \\
0.806 \\
0.801 \\
0.796 \\
0.789\end{array}$ & $\begin{array}{r}11.76 \\
10.99 \\
10.63 \\
10.28 \\
9.96 \\
9.65 \\
9.35 \\
13.49 \\
12.63 \\
12.23 \\
11.85 \\
11.04 \\
11.94 \\
10.81\end{array}$ & $\begin{array}{l}0.505 \\
0.498 \\
0.496 \\
0.494 \\
0.494 \\
0.487 \\
0.482 \\
0.511 \\
0.504 \\
0.509 \\
0.459 \\
0.496 \\
0.494 \\
0.489\end{array}$ & $\begin{array}{l}0.312 \\
0.308 \\
0.306 \\
0.305 \\
0.305 \\
0.300 \\
0.297 \\
0.316 \\
0.314 \\
0.3308 \\
0.308 \\
0.306 \\
0.305 \\
0.302\end{array}$ & $\begin{array}{l}38.17 \\
38.17 \\
38.17 \\
38.17 \\
38.17 \\
38.17 \\
38.17 \\
38.17 \\
38.17 \\
38.17 \\
38.17 \\
38.97 \\
38.197 \\
38.19\end{array}$ & $\begin{array}{l}0.208 \\
0.205 \\
0.254 \\
0.203 \\
0.2233 \\
0.230 \\
0.198 \\
0.211 \\
0.207 \\
0.206 \\
0.205 \\
0.204 \\
0.203 \\
0.201\end{array}$ & $\begin{array}{l}0.104 \\
0.103 \\
0.102 \\
0.102 \\
0.102 \\
0.100 \\
0.099 \\
0.105 \\
0.104 \\
0.103 \\
0.1103 \\
0.102 \\
0.102 \\
0.101\end{array}$ & $\begin{array}{l}3.215 \\
3.225 \\
3.227 \\
3.222 \\
3.292 \\
3.323 \\
3.310 \\
3.212 \\
3.223 \\
3.225 \\
3.228 \\
3.220 \\
3.293 \\
3.236\end{array}$ & 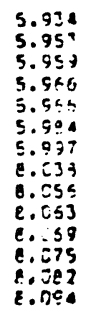 \\
\hline
\end{tabular}

Fig. 19. -Dosificaciones procesadas por computación para agregado redondeado y modulo granulométrico; total 5,50. 


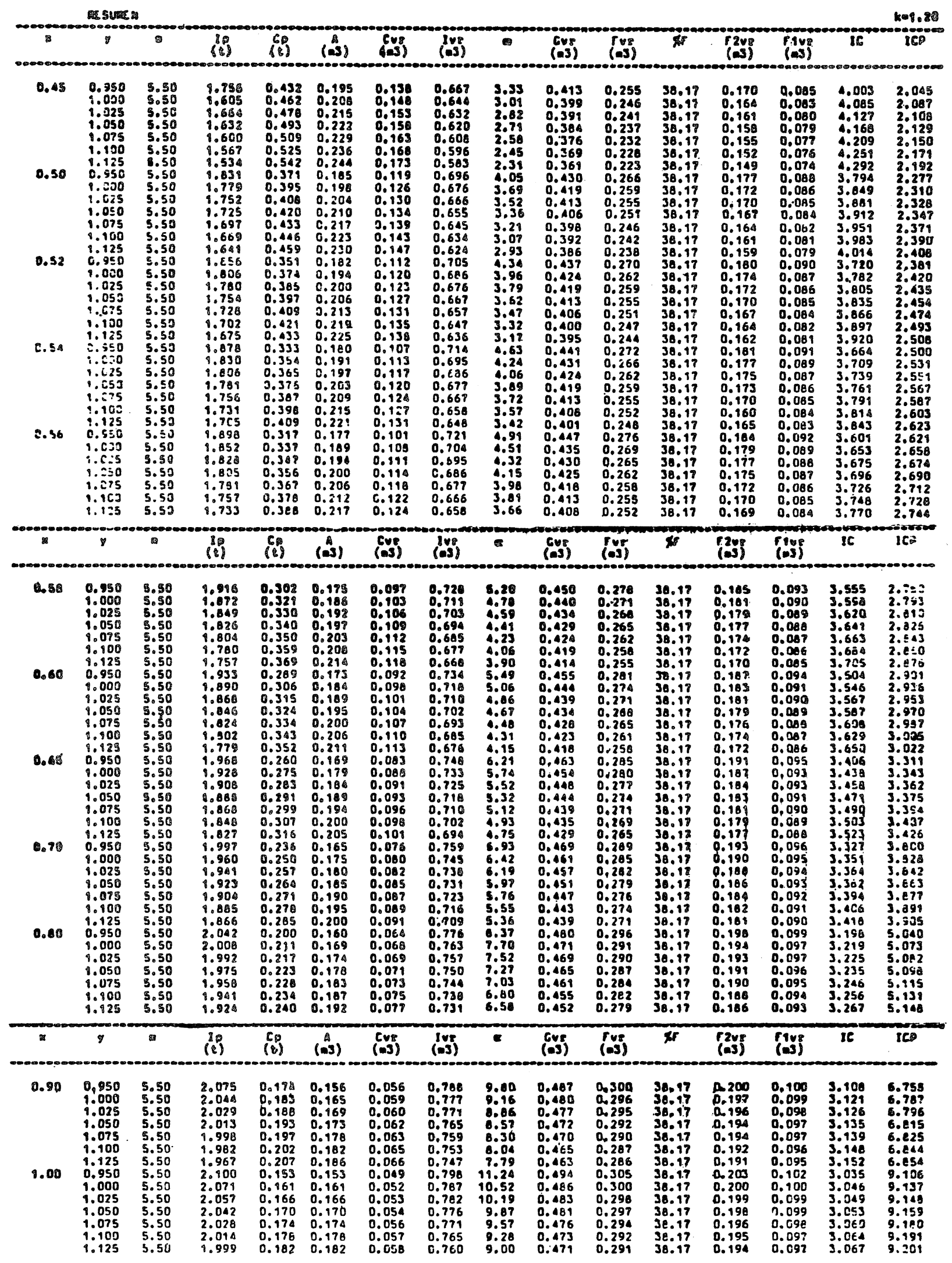

Fig. 20-Dosificaciones procesadas por computación para agregado angular y módulo granulométrico; total 5,50. 